\title{
Continuity properties of Markov semigroups and their restrictions to invariant $L^{1}$-spaces
}

\author{
Sander C. Hille • Daniël T.H. Worm
}

Received: 30 March 2009 / Accepted: 10 July 2009 / Published online: 27 August 2009

(C) The Author(s) 2009. This article is published with open access at Springerlink.com

\begin{abstract}
We consider Markov semigroups on the cone of positive finite measures on a complete separable metric space. Such a semigroup extends to a semigroup of linear operators on the vector space of measures that typically fails to be strongly continuous for the total variation norm. First we characterise when the restriction of a Markov semigroup to an invariant $L^{1}$-space is strongly continuous. Aided by this result we provide several characterisations of the subspace of strong continuity for the total variation norm. We prove that this subspace is a projection band in the Banach lattice of finite measures, and consequently obtain a direct sum decomposition.
\end{abstract}

Keywords Markov semigroups $\cdot$ Strong continuity $\cdot$ Invariant subspaces

\section{Introduction}

Markov operators on the cone of positive finite measures are additive and positively homogeneous operators on this cone that preserve mass, i.e. the total variation norm of measures. A Markov semigroup is a semigroup of Markov operators. They naturally occur in probability theory and the theory of Markov processes [13, 19]. Moreover, one encounters such semigroups also in the setting of measure-valued structured population models (cf. e.g. [6, 7] and an application to cell growth and division in [5]). Here the measure represents the constitution of the population at each time.

The Markov semigroups that are obtained in both settings are hardly ever continuous for the total variation norm $\|\cdot\|_{\mathrm{TV}}$ on the space of finite measures $\mathcal{M}(S)$ on

Communicated by Rainer Nagel.

S.C. Hille $(\bowtie) \cdot$ D.T.H. Worm

Mathematical Institute, University Leiden, P.O. Box 9512, 2300 RA Leiden, The Netherlands

e-mail: shille@math.leidenuniv.nl

D.T.H. Worm

e-mail: dworm@math.leidenuniv.nl 
the underlying measurable space $(S, \Sigma)$, typically a complete separable metric space with its Borel $\sigma$-algebra. Notable exceptions are Markov jump processes [13, 14], which yield strongly continuous semigroups in $\mathcal{M}(S)$ for $\|\cdot\|_{\mathrm{TV}}$ even when $(S, \Sigma)$ is merely a measurable space as above [29]. This may have motivated other researchers to consider the more restrictive setting of strongly continuous Markov semigroups on $L^{1}$-spaces with respect to particular positive measures (see e.g. [20, 27, 28]). In view of the above mentioned applications this setting seems to be too restrictive however.

In this paper we consider Markov semigroups $(P(t))_{t \geq 0}$ on the positive finite Borel measures $\mathcal{M}^{+}(S)$ on a complete separable metric space $(S, d)$. The positive operators $P(t)$ naturally extend to bounded linear operators $\overline{P(t)}$ on the Banach lattice $\left(\mathcal{M}(S),\|\cdot\|_{\mathrm{TV}}\right)$. We address two closely related questions. In the case that $(P(t))_{t \geq 0}$ leaves invariant a cone $\Gamma \subset \mathcal{M}^{+}(S)$ such that the measures in $\Gamma$ are all absolutely continuous with respect to a single measure $\mu$, i.e. $\Gamma=L_{+}^{1}(S, \mu)$, it induces a semigroup of nonexpansive linear operators on $L^{1}(S, \mu)$ that are isometries on $L_{+}^{1}(S, \mu)$. The first question is then to characterise when this induced semigroup is strongly continuous. This is achieved in Theorem 4.6, partially using an argument inspired by [17], under the assumption that for each $\mu \in \mathcal{M}^{+}(S)$, the map $t \mapsto P(t) \mu: \mathbb{R}_{+} \rightarrow \mathcal{M}^{+}(S)$ is continuous for the relative topology on $\mathcal{M}^{+}(S)$ of the weak*-topology on $C_{b}(S)^{*}$. It was shown in $[10,11]$ that this topology is metrisable by means of the norm on $\operatorname{BL}(S)^{*}$, the dual of the bounded Lipschitz functions on $S$. See also [18] for further exploration of this property.

Also under this assumption and the additional assumption that $(P(t))_{t \geq 0}$ is regular (see Definition 3.2), we then deal with the second question in Theorems 5.6 and 5.7, which is to characterise the subspace $\mathcal{M}(S)_{\mathrm{TV}}^{0}$ of $\mathcal{M}(S)$ that consists of all measures $\mu$ that are continuous $\left(C^{0}\right)$ for the total variation norm topology, i.e. all $\mu$ for which $t \mapsto P(t) \mu$ is continuous for $\|\cdot\|_{\mathrm{TV}}$. This subspace contains in particular all invariant measures. The characterisation exploits results of [15] on modules of Banach algebras with approximate identity and properties of Bochner integration in the Banach space $\mathcal{S}_{\mathrm{BL}}$, which is the closure of $\mathcal{M}(S)$ in $\mathrm{BL}(S)^{*}$. These properties are of separate interest. We state and prove them in Sect. 2.3. A consequence of the characterisation is that $\mathcal{M}(S)_{\mathrm{TV}}^{0}$ is dense in $\mathcal{M}(S)$ for the $\mathcal{S}_{\mathrm{BL}}$-topology. In particular it is non-trivial and not 'too small'. Moreover, it turns out to be a projection band in the Banach lattice $\mathcal{M}(S)$ (Proposition 6.1), hence it is complemented. This complement is characterised and will not be $(P(t))_{t \geq 0}$-invariant in general (unfortunately). An additional result of our approach is a generalisation of a classical result by Wiener and Young [30] for general Markov semigroups (Theorem 6.7).

We start with a particular metric $d$, rather than the setting of a Polish space, because we want to view the restriction of the weak*-topology of $C_{b}(S)^{*}$ to $\mathcal{M}^{+}(S)$ as induced by a norm: the dual norm in $\operatorname{BL}(S)^{*}$ which seems to depend on the metric. Apparently, many properties we prove depend only on the topology generated by the metric. However, formulation in terms of an 'enveloping' Banach space may be beneficial when considering perturbation theory of Markov semigroups, because one can then apply the abundance of available results in the literature, instead of having to consider perturbation theory in the setting of locally convex topological vector spaces. It may be interesting to further investigate the impact of changes in the metric though. 


\subsection{Some notational conventions}

We write $(\Omega, \Sigma)$ to denote a measurable space, $\mathcal{M}^{+}(\Omega)$ to denote the cone of positive finite measures on $\Sigma, \mathcal{M}(\Omega)$ the real vector space of all signed finite measures and $\operatorname{BM}(\Omega)$ the real vector space of all bounded measurable functions from $\Omega$ to $\mathbb{R}$. Throughout this paper $(S, d)$ will denote a complete separable metric space, viewed as a measurable space with respect to its Borel $\sigma$-algebra, with at least two elements. We write $\mathbb{1}_{E}$ for the indicator function of $E \subset S . \mathbb{1}_{S}$ will be simplified to $\mathbb{1}$. For $f: \Omega \rightarrow \mathbb{R}$ measurable and $\mu \in \mathcal{M}(\Omega)$ we write $\langle\mu, f\rangle$ for $\int_{\Omega} f d \mu$. In the sequel we use 'subscript BL' to denote the space being equipped with the relative topology of $\mathrm{BL}(S)^{*}$ (see Sect. 2.2) and 'subscript TV' to designate the total variation norm topology.

\section{Preliminaries on spaces of measures}

$\mathcal{M}(\Omega)$ endowed with the total variation norm $\|\cdot\|_{\mathrm{TV}}$ is a Banach space. Let $\mu, v \in$ $\mathcal{M}(\Omega) . \mu$ is absolutely continuous with respect to $\nu, \mu \ll \nu$, if $|\mu|(E)=0$ for every $E \in \Sigma$ for which $|\nu|(E)=0$.

Let $\mu \in \mathcal{M}(\Omega), v \in \mathcal{M}^{+}(\Omega)$, then $\mu \ll v$ if and only if $\mu(E)=0$ for every $E \in \Sigma$ such that $\nu(E)=0$, which is easy to prove.

Lemma 2.1 Let $\mu \in \mathcal{M}(S), v \in \mathcal{M}^{+}(S)$. Then the following are equivalent:

(i) $\mu \ll v$

(ii) $\mu(K)=0$ for all compact $K$ in $S$ such that $\nu(K)=0$.

Proof (i) $\Rightarrow$ (ii): Trivial. (ii) $\Rightarrow$ (i): Let $E$ be a Borel set in $S$ such that $\nu(E)=0$. Then $\nu(K)=0$ for all compact $K$ such that $K \subset E$, hence $\mu^{+}(K)=\mu^{-}(K)$ for all compact $K \subset E$. Since $S$ is a complete separable metric space, $\mu^{+}$and $\mu^{-}$ are inner regular, i.e. for every Borel set $E$ in $S$, there are compact $K_{n} \subset E$, such that $\lim _{n \rightarrow \infty} \mu^{+}\left(K_{n}\right)=\mu^{+}(E)$ and $\lim _{n \rightarrow \infty} \mu^{-}\left(K_{n}\right)=\mu^{-}(E)$ (see e.g. [3, Theorems 1.1 and 1.3]). So $\mu^{+}(E)=\mu^{-}(E)$ and $\mu(E)=\mu^{+}(E)-\mu^{-}(E)=0$.

\subsection{Space of measures viewed as Banach lattice}

We refer to $[2,22,32]$ for the basic theory on Riesz spaces and Banach lattices.

$\mathcal{M}(\Omega)$ is an ordered vector space for the partial ordering defined by

$$
\mu \leq v \text { whenever } \mu(E) \leq v(E) \text { for all } E \in \Sigma \text {. }
$$

$\mathcal{M}(\Omega)$ is a Riesz space, where the least upper bound of $\mu$ and $v$ is given by

$$
\mu \vee v(E):=\sup \{\mu(A)+v(E \backslash A) \mid A \in \Sigma, A \subset E\},
$$

and the greatest lower bound is given by

$$
\mu \wedge \nu(E):=\inf \{\mu(A)+v(E \backslash A) \mid A \in \Sigma, A \subset E\} .
$$


Note that $|\mu| \leq|\nu|$ implies $\mu \ll \nu$. The positive and negative part of $\mu \in \mathcal{M}(\Omega)$ as introduced in measure theory, $\mu^{+}$and $\mu^{-}$, correspond to the concepts of positive and negative part in a Riesz space: $\mu^{+}=\mu \vee 0, \mu^{-}=(-\mu)^{+}$and $|\mu|=\mu^{+}+\mu^{-}$. $\mu, v \in \mathcal{M}(\Omega)$ are mutually singular, $\mu \perp v$, if there is a $U \in \Sigma$, such that $\mu(E)=$ $\mu(E \cap U)$ and $v(E)=v(E \backslash U)$ for every $E \in \Sigma$. Mutual singularity of $\mu, v \in \mathcal{M}(\Omega)$ corresponds to the concept of disjointness in a Riesz space: $\mu$ and $\nu$ are disjoint, $\mu \perp \nu$, whenever $|\mu| \wedge|\nu|=0 . \mathcal{M}(\Omega)$ is a Dedekind complete Riesz space [22, 1.1 Example vi].

$\mathcal{M}(\Omega)$ is a Banach lattice for the total variation norm: $\|\mu\|_{\mathrm{TV}}=|\mu|(\Omega)$, and $\|\cdot\|_{\mathrm{TV}}$ is an $L$-norm: $\|\mu+v\|_{\mathrm{TV}}=\|\mu\|_{\mathrm{TV}}+\|v\|_{\mathrm{TV}}$ for all $\mu, v \in \mathcal{M}^{+}(\Omega)$, hence $\mathcal{M}(\Omega)$ is an $L$-space. This also implies that $\|\mu+v\|_{\mathrm{TV}}=\|\mu\|_{\mathrm{TV}}+\|v\|_{\mathrm{TV}}$ for all $\mu, v \in \mathcal{M}(\Omega)$, such that $\mu \perp v$. As in all Banach lattices, the lattice operations are continuous for the norm topology (see e.g. [22, Proposition 1.1.6]).

We will now recall some concepts in Riesz spaces that we will need later on: Let $X$ be a Riesz space. A subspace $I$ of $X$ is an ideal of $X$ if $|x| \leq|y|$ for some $y \in I$ implies $x \in I$. An ideal $B$ of $X$ is a band of $X$ if $\sup (A) \in B$ for every subset $A \subset B$ which has a supremum in $X$. A band $B$ of $X$ is a projection band if there exists a bounded linear projection $P: X \rightarrow B$, such that $0 \leq P x \leq x$ for all $x \in X_{+}$. In this case $X=B \oplus B^{\perp}$, where $B^{\perp}:=\{x \in X: x \perp y$ for all $y \in B\}$.

In a remark in [2] (under Definition 4.20) it is shown that every $L$-space has order continuous norm as a consequence of [22, Theorem 2.4.2]. Furthermore, in a Banach lattice with order continuous norm, every closed ideal is a projection band [22, Corollary 2.4.2]. These statements imply

Theorem 2.2 Every closed ideal in $\mathcal{M}(\Omega)$ is a projection band.

\subsection{The space $\mathcal{S}_{\mathrm{BL}}$}

In this section we recall some definitions and results from [18]. $\operatorname{BL}(S)$ denotes the Banach space of bounded real-valued Lipschitz functions for the metric $d$, endowed with the norm $\|f\|_{\mathrm{BL}}:=|f|_{\text {Lip }}+\|f\|_{\infty}$, where

$$
|f|_{\text {Lip }}:=\sup \left\{\frac{|f(x)-f(y)|}{d(x, y)}: x, y \in S, x \neq y\right\} .
$$

The Dirac functionals $\delta_{x}(f):=f(x)$ for $x \in S$ are in $\operatorname{BL}(S)^{*}$. We denote the usual dual norm on $\mathrm{BL}(S)^{*}$ by $\|\cdot\|_{\mathrm{BL}}^{*}$.

$\mathrm{BL}(S)$ is in fact isometrically isomorphic to the dual of a separable Banach space $\mathcal{S}_{\mathrm{BL}}$, which can be defined as the closure of the finite linear span of the $\delta_{x}, x \in S$, in $\operatorname{BL}(S)^{*}$. A function $f \in \operatorname{BL}(S)$ defines a bounded linear functional on $\mathcal{S}_{\mathrm{BL}}$ by sending $\phi$ to $\phi(f)$. Then, as shown in [10, Lemma 6], each $\mu \in \mathcal{M}(S)$ defines a unique element in $\operatorname{BL}(S)^{*}$, which we will also denote by $\mu$, by sending $f \in \operatorname{BL}(S)$ to $\langle\mu, f\rangle=\int_{S} f d \mu$. Using [18, Lemma 3.5] one can show that the map $x \mapsto \delta_{x}$ is a continuous embedding from $S$ into $\mathcal{S}_{\mathrm{BL}}$.

By [18, Theorem 3.9 and Corollary 3.10], $\mathcal{M}^{+}(S)$ is a $\|\cdot\|_{\mathrm{BL}}^{*}$-closed convex cone of $\mathcal{S}_{\mathrm{BL}}$, and $\mathcal{M}(S)$ is a $\|\cdot\|_{\mathrm{BL}}^{*}$-dense subspace of $\mathcal{S}_{\mathrm{BL}}$. 
The restriction of the weak-star topology on $C_{b}(S)^{*}$ to $\mathcal{M}^{+}(S)$ equals the restriction of the norm topology on $\mathcal{S}_{\mathrm{BL}}$ to $\mathcal{M}^{+}(S)$ by [10, Theorem 18], in particular the following lemma holds:

Lemma 2.3 Let $\mu_{n}, \mu \in \mathcal{M}^{+}(S)$. Then $\left\|\mu_{n}-\mu\right\|_{\mathrm{BL}}^{*} \rightarrow 0$ if and only if $\int_{S} f d \mu_{n} \rightarrow$ $\int_{S} f d \mu$ for all $f \in C_{b}(S)$.

Let

$$
\mathcal{S}_{\mathrm{BL}}^{+}:=\left\{\phi \in \mathcal{S}_{\mathrm{BL}}: \phi(f) \geq 0 \text { for all } f \in \mathrm{BL}(S), f \geq 0\right\} .
$$

Then $\mathcal{S}_{\mathrm{BL}}^{+}=\mathcal{M}^{+}(S)$ by [18, Corollary 4.2].

When $\mathcal{M}(S)$ and $\mathcal{M}^{+}(S)$ are equipped with the $\|\cdot\|_{\mathrm{BL}}^{*}$-topology, we write $\mathcal{M}(S)_{\mathrm{BL}}$ and $\mathcal{M}^{+}(S)_{\mathrm{BL}}$ respectively. When we use the $\|\cdot\|_{\mathrm{TV}}$-topology, we write $\mathcal{M}(S)_{\mathrm{TV}}$ and $\mathcal{M}^{+}(S)_{\mathrm{TV}}$.

By [18, Lemma 3.1 and Eq. (3)], $\|\mu\|_{\mathrm{TV}} \leq\|\mu\|_{\mathrm{BL}}^{*}$ for every $\mu \in \mathcal{M}(S)$, with equality whenever $\mu \in \mathcal{M}^{+}(S)$, thus $\mathcal{M}(S)_{\mathrm{TV}}$ embeds continuously into $\mathcal{M}(S)_{\mathrm{BL}}$.

\subsection{Bochner integration of $\mathcal{S}_{\mathrm{BL}}$-valued functions}

In this section we give some results on functions $p: \Omega \rightarrow \mathcal{S}_{\mathrm{BL}}^{+}$which are strongly measurable in the sense of Bochner. We will make use of the Monotone Class Theorem for functions, which we state here for convenience (see e.g. [31, Theorem II.4]).

Theorem 2.4 Let $\mathcal{E}$ be a $\pi$-system for $S$ and let $\mathcal{H}$ be a vector space of functions from $S$ to $\mathbb{R}$ such that

1. $\mathcal{H}$ contains the indicator function $\mathbb{1}_{E}$ of every $E \in \mathcal{E}$, and $\mathcal{H}$ contains $\mathbb{1}_{S}$

2. if $\left(f_{n}\right)_{n}$ is a sequence of elements of $\mathcal{H}$ with $f_{n} \geq 0$ and $f_{n} \uparrow f$, where $f$ is bounded, then $f \in \mathcal{H}$.

Then $\mathcal{H}$ contains every bounded real-valued function which is measurable with respect to the $\sigma$-algebra generated by $\mathcal{E}$.

Proposition 2.5 Let $p: \Omega \rightarrow \mathcal{S}_{\mathrm{BL}}^{+}$. Then the following conditions are equivalent:

(i) $p$ is strongly measurable

(ii) for each bounded measurable $f: S \rightarrow \mathbb{R}$, the map $\Omega \rightarrow \mathbb{R}: \omega \mapsto\langle p(\omega), f\rangle$ is measurable

(iii) for each Borel measurable $E \subset S$, the map $\Omega \rightarrow \mathbb{R}: \omega \mapsto p(\omega)(E)$ is measurable from $\Omega$ to $\mathbb{R}$.

Proof (i) $\Rightarrow$ (ii): Let $\mathcal{H}$ the vector space of measurable functions $h$ from $S$ to $\mathbb{R}$, such that $\omega \mapsto\langle p(\omega), h\rangle$ is measurable from $\Omega$ to $\mathbb{R}$. Let $\mathcal{C}$ be the $\pi$-system of closed sets in $S$. Our aim is to show that $\mathcal{H}$ and $\mathcal{C}$ satisfy the conditions of Theorem 2.4. Then it follows that $\mathcal{H}$ contains every bounded Borel measurable function on $S$.

Since $p$ is strongly measurable, it is weakly measurable. Let $C$ be a closed set in $S$ and let $g_{n}(x):=\max (1-n d(x, C), 0)$. Then $g_{n} \in \mathrm{BL}(S) \cong \mathcal{S}_{\mathrm{BL}}^{*}$, hence $G_{n}: \omega \mapsto$ 
$\left\langle p(\omega), g_{n}\right\rangle$ is measurable from $\Omega$ to $\mathbb{R}$. Since $C$ is closed, $g_{n}(x) \rightarrow \mathbb{1}_{C}(x)$ for every $x \in S$. Fix $\omega \in \Omega$. Then all $g_{n}$ are in $L^{1}(p(\omega))$, thus $\mathbb{1}_{C}$ is in $L^{1}(p(\omega))$ and

$$
\lim _{n \rightarrow \infty} G_{n}(\omega)=\left\langle p(\omega), \mathbb{1}_{C}\right\rangle
$$

by the Lebesgue Dominated Convergence Theorem. So the function

$$
\omega \rightarrow p(\omega)(C)=\left\langle p(\omega), \mathbb{1}_{C}\right\rangle
$$

is the pointwise limit of measurable functions, hence measurable, which implies that $\mathbb{1}_{C} \in \mathcal{H}$ for all closed $C \subset S$. Suppose $h_{n} \in \mathcal{H}$ such that $0 \leq h_{n} \uparrow h \leq M$, for some function $h: \Omega \rightarrow \mathbb{R}$, bounded by $M>0$. Then by assumption $H_{n}: \omega \mapsto\left\langle p(\omega), h_{n}\right\rangle$ is measurable for all $n \in \mathbb{N}$. Fix $\omega \in \Omega$. By the Lebesgue Monotone Convergence Theorem $h \in L^{1}(p(\omega))$ and $\lim _{n \rightarrow \infty} H_{n}(\omega)=\langle p(\omega), h\rangle$. This implies that the function $\omega \mapsto\langle p(\omega), h\rangle$ is the pointwise limit of measurable functions, hence measurable. So $h \in \mathcal{H}$ and the conditions of Theorem 2.4 are satisfied.

(ii) $\Rightarrow$ (iii): Let $E \subset S$ be measurable, then $\mathbb{1}_{E}$ is a bounded measurable function from $S$ to $\mathbb{R}$.

(iii) $\Rightarrow$ (i): By assumption $\omega \mapsto\langle p(\omega), g\rangle$ is measurable, for all simple functions $g$ on $S$. Let $h \in \mathrm{BL}^{+}(S)$. Then there are simple functions $h_{n}$ such that $0 \leq h_{n} \uparrow h$. By the Lebesgue Monotone Convergence Theorem, $\left\langle p(\omega), h_{n}\right\rangle \rightarrow\langle p(\omega), h\rangle$ for every $\omega \in \Omega$. So $\omega \mapsto\langle p(\omega), h\rangle$ is the pointwise limit of measurable functions, hence measurable. For general $h \in \operatorname{BL}(S)$, we can write $h=h^{+}-h^{-}$, and thus $\omega \mapsto\langle p(\omega), h\rangle$ is the difference of two measurable functions, hence measurable. So $p$ is weakly measurable. Since $\mathcal{S}_{\mathrm{BL}}$ is separable, $p$ is strongly measurable by Pettis' Theorem.

If $p: \Omega \rightarrow \mathcal{S}_{\mathrm{BL}}^{+}$is Bochner integrable with respect to $\mu \in \mathcal{M}^{+}(\Omega)$, then $v:=$ $\int_{\Omega} p(\omega) d \mu(\omega)$ defines an element in $\mathcal{S}_{\mathrm{BL}}$. Since $\mathcal{S}_{\mathrm{BL}}^{+}=\mathcal{M}^{+}(S)$ is a closed convex cone in $\mathcal{S}_{\mathrm{BL}}, v$ is in $\mathcal{S}_{\mathrm{BL}}^{+}$.

Proposition 2.6 Let $p: \Omega \rightarrow \mathcal{S}_{\mathrm{BL}}^{+}$be Bochner integrable with respect to $\mu$ in $\mathcal{M}^{+}(\Omega)$, and define $v:=\int_{\Omega} p(\omega) d \mu(\omega)$. Then

$$
\int_{S} f d \nu=\int_{\Omega}\langle p(\omega), f\rangle d \mu(\omega),
$$

for any bounded measurable $f: S \rightarrow \mathbb{R}$.

Proof Step 1. (1) holds for all $f \in \mathrm{BL}(S)$.

We can view $f$ as element of $\mathcal{S}_{\mathrm{BL}}^{*}$. Since $p$ is Bochner integrable with respect to $\mu$, the map $\Omega \rightarrow \mathbb{R}: \omega \mapsto\langle p(\omega), f\rangle$ is in $L^{1}(\mu)$. So we get by [8, Theorem 6] that

$$
\begin{aligned}
\int_{S} f d v=\langle\nu, f\rangle & =\left\langle\int_{\Omega} p(\omega) d \mu(\omega), f\right\rangle \\
& =\int_{\Omega}\langle p(\omega), f\rangle d \mu(\omega) .
\end{aligned}
$$


Step 2. (1) holds for all $f=\mathbb{1}_{C}, C \subset S$ closed.

Let $f_{n} \in \operatorname{BL}(S)$ be defined as $f_{n}(x):=\max (1-n d(x, C), 0)$. Then $f_{n}$ is bounded by $\mathbb{1}$, and $f_{n}(x) \rightarrow \mathbb{1}_{C}(x)$ for all $x \in S$, so by Lebesgue Dominated Convergence Theorem we have that for all $\omega \in \Omega,\left\langle p(\omega), f_{n}\right\rangle \rightarrow\left\langle p(\omega), \mathbb{1}_{C}\right\rangle=[p(\omega)](C)$. Since $f_{n} \in \mathcal{S}_{\mathrm{BL}}^{*}, \omega \mapsto\left\langle p(\omega), f_{n}\right\rangle$ is in $L^{1}(\mu)$. Also

$$
\left\|\left\langle p(\omega), f_{n}\right\rangle\right\| \leq\|p(\omega)\|_{\mathrm{TV}}=\|p(\omega)\|_{\mathrm{BL}}^{*},
$$

for all $\omega \in \Omega$ and $n \in \mathbb{N}$, and by assumption $\omega \mapsto\|p(\omega)\|_{\mathrm{BL}}^{*}$ is in $L^{1}(\mu)$. Hence by the Lebesgue Dominated Convergence Theorem

$$
\int_{\Omega}\left\langle p(\omega), f_{n}\right\rangle d \mu(\omega) \rightarrow \int_{\Omega}[p(\omega)](C) d \mu(\omega) .
$$

By Step 1

$$
\int_{\Omega}\left\langle p(\omega), f_{n}\right\rangle d \mu(\omega)=\int_{S} f_{n} d \nu,
$$

for all $n \in \mathbb{N}$. And again by the Lebesgue Dominated Convergence Theorem we can conclude that $\int_{S} f_{n} d \nu \rightarrow v(C)$. So $\int_{\Omega}[p(\omega)](C) d \mu(\omega)=v(C)$ for all $C$ closed.

Step 3. (1) holds for all bounded measurable $f: S \rightarrow \mathbb{R}$.

Now we want to apply Theorem 2.4 . Let $\mathcal{H}$ be the vector space of bounded measurable functions $f: S \rightarrow \mathbb{R}$, such that $\int_{\Omega}\langle p(\omega), f\rangle d \mu(\omega)=\int_{S} f d \nu$. Note that these expressions are well defined: $f$ is bounded and measurable, so it follows from Proposition 2.5 that $\Omega \rightarrow \mathbb{R}: \omega \mapsto\langle p(\omega), f\rangle$ is in $L^{1}(\mu)$.

By Step $2 \mathbb{1}_{C} \in \mathcal{H}$ for all $C \subset S$ closed. Now let $f_{n} \in \mathcal{H}$ with $0 \leq f_{n} \uparrow f \leq$ $M<\infty$, for some function $f$ and some $M>0$. Then by the Lebesgue Monotone Convergence Theorem, $\left\langle p(\omega), f_{n}\right\rangle \rightarrow\langle p(\omega), f\rangle$ for all $\omega \in \Omega$, and $\int_{S} f_{n} d \nu \rightarrow$ $\int_{S} f d \nu$. Since $\left\langle p(\omega), f_{n}\right\rangle$ is bounded from above by a constant not depending on $n$ and $\omega$, we can apply the Lebesgue Dominated Convergence Theorem to get that

$$
\int_{\Omega}\left\langle p(\omega), f_{n}\right\rangle d \mu(\omega) \rightarrow \int_{\Omega}\langle p(\omega), f\rangle d \mu(\omega)
$$

Since $f_{n} \in \mathcal{H}$ we can conclude that $\int_{\Omega}\langle p(\omega), f\rangle d \mu(\omega)=\int_{S} f d \nu$, hence $f \in \mathcal{H}$. By Theorem 2.4 we obtain that $\mathcal{H}$ contains every bounded real-valued Borel measurable function.

Corollary 2.7 Let $p: \Omega \rightarrow \mathcal{S}_{\mathrm{BL}}^{+}$be Bochner integrable with respect to $\mu$ in $\mathcal{M}^{+}(\Omega)$. Then

$$
\left[\int_{\Omega} p(\omega) d \mu(\omega)\right](E)=\int_{\Omega} p(\omega)(E) d \mu(\omega)
$$

for any Borel measurable $E \subset S$.

\section{Markov semigroups}

We start by introducing the concept of Markov operators. 
Definition 3.1 A Markov operator is a map $P: \mathcal{M}^{+}(S) \rightarrow \mathcal{M}^{+}(S)$, such that

(MO1) $P$ is additive and $\mathbb{R}_{+}$-homogeneous

(MO2) $\|P \mu\|_{\mathrm{TV}}=\|\mu\|_{\mathrm{TV}}$ for all $\mu \in \mathcal{M}^{+}(S)$.

Since $\mathcal{M}(S)_{\mathrm{TV}}$ is a Banach lattice, condition (MO1) ensures that a Markov operator $P$ extends to a positive bounded linear operator on $\mathcal{M}(S)_{\mathrm{TV}}$ given by $P \mu:=P\left(\mu^{+}\right)-P\left(\mu^{-}\right)$. The operator norm of this extension is

$$
\|P\|=\sup \left\{\|P \mu\|_{\mathrm{TV}}: \mu \in \mathcal{M}^{+}(S),\|\mu\|_{\mathrm{TV}} \leq 1\right\}=1
$$

according to $(\mathrm{MO} 2)$. Since $\mathrm{Id}: \mathcal{M}(S)_{\mathrm{TV}} \rightarrow \mathcal{M}(S)_{\mathrm{BL}}$ is continuous with operator norm equal to $1,(\mathrm{MO} 2)$ implies that $P: \mathcal{M}(S)_{\mathrm{TV}} \rightarrow \mathcal{M}(S)_{\mathrm{BL}}$ is nonexpansive and an isometry on the positive cone.

Definition 3.2 A Markov operator $P$ is regular if there exists an $U: \operatorname{BM}(S) \rightarrow$ $\mathrm{BM}(S)$, called the dual of $P$, such that

$$
\langle P \mu, f\rangle=\langle\mu, U f\rangle,
$$

for all $\mu \in \mathcal{M}(\Omega), f \in \mathrm{BM}(\Omega)$.

We will show that a continuity property on $P$ ensures regularity:

Lemma 3.3 If $P$ is a Markov operator such that $P: \mathcal{M}^{+}(S)_{\mathrm{BL}} \rightarrow \mathcal{M}^{+}(S)_{\mathrm{BL}}$ is continuous, then $P$ is regular and the dual $U$ leaves $C_{b}(S)$ invariant.

Proof For $f \in \operatorname{BM}(S)$ define $U f(x):=\left\langle P \delta_{x}, f\right\rangle, x \in S$. Since $x \mapsto \delta_{x}$ is a continuous embedding from $S$ into $\mathcal{S}_{\mathrm{BL}}^{+}=\mathcal{M}^{+}(S)_{\mathrm{BL}}, x \mapsto P \delta_{x}$ is also continuous, hence strongly measurable, from $S$ to $\mathcal{S}_{\mathrm{BL}}^{+}$. So by Proposition 2.5 the map $x \mapsto\left\langle P \delta_{x}, f\right\rangle$ is measurable. It is also bounded by (MO2) and boundedness of $f$. So $U$ maps $\operatorname{BM}(S)$ into itself. Let $g \in C_{b}(S) \subset \mathrm{BM}(S)$. Using Lemma 2.3 and continuity of $P$, it can be shown that $x \mapsto\left\langle P \delta_{x}, g\right\rangle$ is continuous from $S$ to $\mathbb{R}$, hence $U g \in C_{b}(S)$.

By linearity it suffices to show (2) for $\mu \in \mathcal{M}^{+}(S), f \in \mathrm{BM}(S)$. By Proposition 2.6

$$
\langle\mu, U f\rangle=\int_{S}\left\langle P \delta_{x}, f\right\rangle d \mu(x)=\int_{S} f d \tilde{P} \mu,
$$

where $\tilde{P} \mu:=\int_{S} P \delta_{x} d \mu(x)$. Now it remains to prove that $\tilde{P} \mu=P \mu$. Clearly $\tilde{P}$ is positively homogeneous and additive from $\mathcal{M}^{+}(S)$ to $\mathcal{M}^{+}(S)$. Also, $\tilde{P} \delta_{y}=P \delta_{y}$ for all $y \in S$. So $P$ and $\tilde{P}$ coincide on

$$
D^{+}:=\left\{\sum_{i=1}^{n} \alpha_{i} \delta_{x_{i}}: n \in \mathbb{N}, \alpha_{i} \in \mathbb{R}_{+}, x_{i} \in S\right\},
$$

which is dense in $\mathcal{S}_{\mathrm{BL}}^{+}$. Let $\mu_{n} \rightarrow \mu$ in $\mathcal{S}_{\mathrm{BL}}^{+}$, then for all $g \in C_{b}(S)$,

$$
\int_{S} g d \tilde{P} \mu_{n}=\left\langle\mu_{n}, U g\right\rangle \rightarrow\langle\mu, U g\rangle=\int_{S} g d \tilde{P} \mu,
$$


by Lemma 2.3 , thus $\tilde{P}$ is also continuous from $\mathcal{S}_{\mathrm{BL}}^{+}$to $\mathcal{S}_{\mathrm{BL}}^{+}$. Hence $P=\tilde{P}$ on $\mathcal{M}^{+}(S)$ by density of $D^{+}$in $\mathcal{S}_{\mathrm{BL}}^{+}$.

A Markov semigroup is a semigroup $(P(t))_{t \geq 0}$ of Markov operators. $(P(t))_{t \geq 0}$ is called regular if $P(t)$ is regular for all $t \geq 0$.

Remark 3.4 It follows from [19, Proposition 2.4] that a Markov semigroup $(P(t))_{t \geq 0}$ is regular if and only if there exists a Markov transition function $K$ [19, Definition 2.2] such that $P(t) \mu(E)=\int_{S} K(t, x, E) d \mu(x)$ for all $\mu \in \mathcal{M}^{+}(S)$ and $E \subset S$ Borel. Since time-homogeneous Markov processes can be defined using Markov transition functions [13, Chap. 4, Sect. 1], Markov semigroups arising from such processes are regular.

While strong continuity of $(P(t))_{t \geq 0}$ with respect to $\|\cdot\|_{\mathrm{TV}}$ is rare, we will see that strong continuity with respect to $\|\cdot\|_{\mathrm{BL}}^{*}$ is not.

We call the Markov semigroup $(P(t))_{t \geq 0}$ strongly stochastically continuous, when $t \mapsto\langle P(t) \mu, f\rangle$ is continuous for all $\mu \in \mathcal{M}^{+}(S)$ and $f \in C_{b}(S)$.

Lemma 3.5 Let $(P(t))_{t \geq 0}$ be a Markov semigroup. Then the following are equivalent:

(i) $(P(t))_{t \geq 0}$ is strongly stochastically continuous

(ii) $t \mapsto\langle P(t) \mu, f\rangle$ is continuous for all $\mu \in \mathcal{M}(S)$ and $f \in C_{b}(S)$

(iii) $t \mapsto P(t) \mu$ is continuous from $\mathbb{R}_{+}$to $\mathcal{M}(S)_{\mathrm{BL}}$ for every $\mu \in \mathcal{M}(S)$.

Proof (iii) $\Rightarrow$ (ii): Follows from the decomposition $\mu=\mu^{+}-\mu^{-}$.

(ii) $\Rightarrow$ (iii): For every $\mu \in \mathcal{M}(S), t \mapsto P(t) \mu=P(t) \mu^{+}-P(t) \mu^{-}$is continuous from $\mathbb{R}_{+}$to $\mathcal{S}_{\mathrm{BL}}$ by Lemma 2.3 .

(iii) $\Rightarrow$ (i): Follows from Lemma 2.3.

We will show that certain actions of $\mathbb{R}_{+}$on $S$ provide us with an important class of examples of regular Markov semigroups.

A semigroup of measurable maps on $S$ is a family of maps $\left(\Phi_{t}\right)_{t \geq 0}$, such that $\Phi_{t}: S \rightarrow S$ is measurable, $\Phi_{t} \circ \Phi_{s}=\Phi_{t+s}$ and $\Phi_{0}=\operatorname{Id}_{S}$ for all $s, t \in \mathbb{R}_{+} \cdot\left(\Phi_{t}\right)_{t \geq 0}$ is called strongly continuous if the map $\mathbb{R}_{+} \rightarrow S: t \mapsto \Phi_{t}(x)$ is continuous for all $x \in S$.

A specific class of strongly continuous semigroups of measurable maps on $S$ is given by the so-called jointly continuous semigroups of transformations in $S$, which are semigroups $\left(\Phi_{t}\right)_{t \geq 0}$ of maps $\Phi_{t}: S \rightarrow S$, such that $\mathbb{R}_{+} \times S \rightarrow S:(t, x) \mapsto \Phi_{t}(x)$ is continuous. Properties of such semigroups are being studied in e.g. [9].

Proposition 3.6 Let $\left(\Phi_{t}\right)_{t \geq 0}$ be a semigroup of measurable maps on $S$. Then

(i) $P(t) \mu:=\mu \circ \Phi_{t}^{-1}$ defines a regular Markov semigroup $(P(t))_{t \geq 0}$

(ii) $(P(t))_{t \geq 0}$ is strongly stochastically continuous if and only if $\left(\Phi_{t}\right)_{t \geq 0}$ is strongly continuous

(iii) if $\Phi_{t}$ is continuous, then $P(t): \mathcal{M}^{+}(S)_{\mathrm{BL}} \rightarrow \mathcal{M}^{+}(S)_{\mathrm{BL}}$ is continuous. 
Proof (i) Let $\mu \in \mathcal{M}^{+}(S)$. It is easily verified that $P(t) \mu \in \mathcal{M}^{+}(S), P(t) P(s) \mu=$ $P(t+s) \mu$ for all $s, t \in \mathbb{R}_{+}$and $P(0)=\mathrm{Id}$, and that $(P(t))_{t \geq 0}$ satisfies (MO1)(MO2). For $f \in \operatorname{BM}(S)$, we define $U(t) f:=f \circ \Phi_{t}$. Then $U(t)$ maps $\operatorname{BM}(S)$ to $\operatorname{BM}(S)$. Furthermore, for any $f \in \operatorname{BM}(S)$ and $\mu \in \mathcal{M}(S)$ we have

$$
\langle P(t) \mu, f\rangle=\left\langle\mu \circ \Phi_{t}^{-1}, f\right\rangle=\left\langle\mu, f \circ \Phi_{t}\right\rangle,
$$

hence $(P(t))_{t \geq 0}$ is regular.

(ii) Suppose $\left(\Phi_{t}\right)_{t \geq 0}$ is strongly continuous and let $f \in C_{b}(S)$ and $\mu \in \mathcal{M}^{+}(S)$. Then $f \circ \Phi_{t}(x) \rightarrow f \circ \Phi_{s}(x)$ as $t \rightarrow s$. Also, $\left|f \circ \Phi_{t}(x)\right| \leq\|f\|_{\infty} \mathbb{1} \in L^{1}(\mu)$, hence by (3) and the Lebesgue Dominated Convergence Theorem

$$
\lim _{t \rightarrow s}\langle P(t) \mu, f\rangle=\lim _{t \rightarrow s}\left\langle\mu, f \circ \Phi_{t}\right\rangle=\left\langle\mu, f \circ \Phi_{s}\right\rangle=\langle P(s) \mu, f\rangle .
$$

So $(P(t))_{t \geq 0}$ is strongly stochastically continuous.

Now suppose $(P(t))_{t \geq 0}$ is strongly stochastically continuous and let $s \in \mathbb{R}_{+}$and $x \in S$. Define $f(z):=\min \left(d\left(z, \Phi_{s}(x)\right), 1\right)$. Then $f \in C_{b}(S)$ and

$$
t \mapsto\left\langle P(t) \delta_{x}, f\right\rangle=f \circ \Phi_{t}(x)=\min \left(d\left(\Phi_{t}(x), \Phi_{s}(x)\right), 1\right),
$$

is continuous from $\mathbb{R}_{+}$to $\mathbb{R}$. Therefore, if $t \rightarrow s$, then $d\left(\Phi_{t}(x), \Phi_{s}(x)\right) \rightarrow 0$. So $\left(\Phi_{t}\right)_{t \geq 0}$ is strongly continuous.

(iii) Let $\mu_{n}, \mu \in \mathcal{M}^{+}(S)$ such that $\left\|\mu_{n}-\mu\right\|_{\mathrm{BL}}^{*} \rightarrow 0$ as $n \rightarrow \infty$. Then for all $f \in C_{b}(S), \int_{S} f d \mu_{n} \rightarrow \int_{S} f d \mu$ by Lemma 2.3. Hence

$$
\left\langle P(t) \mu_{n}, f\right\rangle=\left\langle\mu_{n}, f \circ \Phi_{t}\right\rangle \rightarrow\left\langle\mu, f \circ \Phi_{t}\right\rangle=\langle P(t) \mu, f\rangle .
$$

Therefore $\left\|P(t) \mu_{n}-P(t) \mu\right\|_{\mathrm{BL}}^{*} \rightarrow 0$, hence $P(t): \mathcal{M}^{+}(S)_{\mathrm{BL}} \rightarrow \mathcal{M}^{+}(S)_{\mathrm{BL}}$ is continuous.

In [18, Sect. 5] it is shown that if, in addition to the conditions above, the maps $\Phi_{t}: S \rightarrow S$ are Lipschitz, then the Markov semigroup $(P(t))_{t \geq 0}$ can be extended to a semigroup of bounded linear operators $(\overline{P(t)})_{t \geq 0}$ on $\mathcal{S}_{\mathrm{BL}}$. Moreover, $(\overline{P(t)})_{t \geq 0}$ is strongly continuous if $\left(\Phi_{t}\right)_{t \geq 0}$ is strongly continuous and $\lim \sup _{t \downarrow 0}\left|\Phi_{t}\right|_{\text {Lip }}<\infty$.

\section{Restriction to invariant $L^{1}$-spaces}

Let $\mu \in \mathcal{M}^{+}(S)$. For $f \in L^{1}(\mu)$ we define $j_{\mu}(f)=f d \mu$. Then $j_{\mu}$ is a linear map from $L^{1}(\mu)$ into $\mathcal{M}(S)$.

Lemma 4.1 The following properties hold:

(i) $j_{\mu}$ is an isometric embedding of $L^{1}(\mu)$ into $\mathcal{M}(S)_{\mathrm{TV}}$, i.e. $\left\|j_{\mu}(f)\right\|_{\mathrm{TV}}=\|f\|_{1}$ for all $f \in L^{1}(\mu)$

(ii) $j_{\mu}$ is a continuous embedding of $L^{1}(\mu)$ into $\mathcal{S}_{\mathrm{BL}}$, with $\left\|j_{\mu}(f)\right\|_{\mathrm{BL}}^{*}=\|f\|_{1}$ for all $f \in L_{+}^{1}(\mu)$ and $\left\|j_{\mu}(f)\right\|_{\mathrm{BL}}^{*} \leq\|f\|_{1}$ for all $f \in L^{1}(\mu)$. 
The proof is straightforward.

Let $P: \mathcal{M}^{+}(S) \rightarrow \mathcal{M}^{+}(S)$ be a Markov operator. Then the following lemma holds:

Lemma 4.2 If $\mu, v \in \mathcal{M}^{+}(S)$ satisfy $\mu \ll v$, then $P \mu \ll P v$.

Proof There exists $f \in L_{+}^{1}(v)$, such that $j_{v}(f)=\mu$. There are $f_{n} \in L_{+}^{\infty}(\mu)$ with $\left\|f_{n}-f\right\|_{1} \rightarrow 0$. According to Lemma 4.1,

$$
\left\|P j_{v}\left(f_{n}\right)-P j_{v}(f)\right\|_{\mathrm{TV}} \leq\left\|j_{v}\left(f_{n}\right)-j_{v}(f)\right\|_{\mathrm{TV}}=\left\|f_{n}-f\right\|_{1} \rightarrow 0 .
$$

Furthermore, $0 \leq j_{v}\left(f_{n}\right) \leq\left\|f_{n}\right\|_{\infty} v$. Hence by positivity of $P, 0 \leq P j_{v}\left(f_{n}\right) \leq$ $\left\|f_{n}\right\|_{\infty} P v$. Therefore $P j_{v}\left(f_{n}\right) \ll P v$, hence $P j_{v}\left(f_{n}\right) \in L_{+}^{1}(P v)$ for all $n \in \mathbb{N}$. Because $L_{+}^{1}(P v)$ is closed in $\mathcal{M}^{+}(S)_{\mathrm{TV}}, P j_{v}(f) \in L_{+}^{1}(P v)$ as well, thus $P \mu \ll P v$.

Corollary 4.3 $P$ leaves $j_{\mu}\left(L_{+}^{1}(\mu)\right)$ invariant if and only if $P \mu \ll \mu$.

Proof Clearly, if $P$ leaves $j_{\mu}\left(L_{+}^{1}(\mu)\right)$ invariant, then in particular $P \mu \ll \mu$. The proof in the opposite direction follows from Lemma 4.2: if $f \in L_{+}^{1}(\mu)$, then $0 \leq$ $j_{\mu}(f) \ll \mu$, hence $P j_{\mu}(f) \ll P \mu \ll \mu$.

Suppose that $P$ leaves $j_{\mu}\left(L_{+}^{1}(\mu)\right)$ invariant. Then $P$ induces an additive and positively homogeneous map $T: L_{+}^{1}(\mu) \rightarrow L_{+}^{1}(\mu)$ :

$$
T f:=j_{\mu}^{-1} \circ P \circ j_{\mu}(f) .
$$

Because $L^{1}(\mu)$ is a Banach lattice, $T$ extends to a positive bounded linear operator on $L^{1}(\mu)$, which we will also denote by $T$, and $\|T f\|_{1}=\|f\|_{1}$ for every $f \in L_{+}^{1}(\mu)$ by Lemma 4.1 and (MO2). So

$$
\|T\|=\sup \left\{\|T f\|_{1} \mid f \in L_{+}^{1}(\mu),\|f\|_{1} \leq 1\right\}=1 .
$$

$T$ will be called the operator (in $L^{1}(\mu)$ ) induced by $P$.

Crucial in our approach is the following general topological closed graph theorem (cf. [23], (14.1.2), p. 313):

Proposition 4.4 Let $f$ map the topological space $S_{1}$ into the topological space $S_{2}$. If $f$ is closed and $S_{2}$ is compact, then $f$ is continuous.

Moreover, we use the following characterisation of relatively weakly compact subsets of $L^{1}$ (e.g. [1], Theorem 5.2.9, p. 109):

Theorem 4.5 Dunford-Pettis Let $(\Omega, \Sigma, \mu)$ be a $\sigma$-finite measure space. In addition let $\mathcal{F}$ be a bounded set in $L^{1}(\mu)$. Then the following conditions on $\mathcal{F}$ are equivalent:

(i) $\mathcal{F}$ is relatively weakly compact 
(ii) for every sequence $A_{n}$ of disjoint measurable sets

$$
\lim _{n \rightarrow \infty} \sup _{f \in \mathcal{F}} \int_{A_{n}}|f| d \mu=0 .
$$

The fundamental result of this section is:

Theorem 4.6 Let $(P(t))_{t \geq 0}$ be a strongly stochastically continuous Markov semigroup. Let $\mu \in \mathcal{M}^{+}(S)$ be such that $j_{\mu}\left(L_{+}^{1}(\mu)\right)$ is $(P(t))_{t \geq 0}$-invariant. Let $(T(t))_{t \geq 0}$ be the semigroup on $L^{1}(\mu)$ induced by $(P(t))_{t \geq 0}$. Then $(T(t))_{t \geq 0}$ is positive and consists of isometries on $L_{+}^{1}(\mu)$. Furthermore, the following statements are equivalent:

(i) $(T(t))_{t \geq 0}$ is strongly continuous

(ii) the map $t \mapsto P(t) \mu$ is continuous from $\mathbb{R}_{+}$to $\mathcal{M}(S)_{\mathrm{TV}}$

(iii) there exists $\tau>0$ such that for any sequence $A_{n}$ of disjoint Borel measurable subsets of $S$,

$$
\lim _{n \rightarrow \infty} \sup _{0 \leq t \leq \tau} P(t) \mu\left(A_{n}\right)=0
$$

Proof $(T(t))_{t \geq 0}$ is positive and consists of isometries on $L_{+}^{1}(\mu)$ by the remarks above Proposition 4.4.

(i) $\Rightarrow$ (ii). From Lemma 4.1 it follows that for every $s, t \in \mathbb{R}_{+}$

$$
\|P(t) \mu-P(s) \mu\|_{\mathrm{TV}}=\left\|P(t) j_{\mu}(\mathbb{1})-P(s) j_{\mu}(\mathbb{1})\right\|_{\mathrm{TV}}=\|T(t) \mathbb{1}-T(s) \mathbb{1}\|_{1} .
$$

By assumption $t \mapsto T(t) \mathbb{1}$ is continuous from $\mathbb{R}_{+}$to $L^{1}(\mu)$, hence $t \mapsto P(t) \mu$ is continuous from $\mathbb{R}_{+}$to $\mathcal{M}(S)_{\mathrm{TV}}$.

(ii) $\Rightarrow$ (iii). For all $s, t \in \mathbb{R}_{+}$we know by Lemma 4.1 that

$$
\|T(t) \mathbb{1}-T(s) \mathbb{1}\|_{1}=\|P(t) \mu-P(s) \mu\|_{\mathrm{TV}} .
$$

By assumption $t \mapsto P(t) \mu$ is continuous from $\mathbb{R}_{+}$to $\mathcal{M}(S)_{\mathrm{TV}}$, so $t \mapsto T(t) \mathbb{1}$ is continuous from $\mathbb{R}_{+}$to $L^{1}(\mu)$.

Let $\tau>0$. By continuity the partial orbit $\{T(t) \mathbb{1}: 0 \leq t \leq \tau\}$ is norm compact, hence weakly compact. According to Theorem 4.5, for any sequence of disjoint measurable sets $A_{n}$,

$$
0=\lim _{n \rightarrow \infty} \sup _{0 \leq t \leq \tau} \int_{A_{n}}|T(t) \mathbb{1}| d \mu=\lim _{n \rightarrow \infty} \sup _{0 \leq t \leq \tau} P(t) \mu\left(A_{n}\right) .
$$

(iii) $\Rightarrow$ (i). Lemma 4.1, Markov operator property (MO2) and (4) yield that each $T(t)$ is an isometry on $L_{+}^{1}(\mu)$ and $\|T(t)\|=1$ for all $t \geq 0$. We write $L_{w}^{1}$ to denote the space $L^{1}(\mu)$ with the weak topology and $\left(\mathcal{S}_{\mathrm{BL}}\right)_{w}$ to denote $\mathcal{S}_{\mathrm{BL}}$ with the weak topology.

Step 1. $t \mapsto T(t) \mathbb{1}_{E}:[0, \tau] \rightarrow L_{w}^{1}$ is continuous for every measurable $E \subset S$.

Our aim is to show that $t \mapsto T(t) \mathbb{1}_{E}:[0, \tau] \rightarrow L_{w}^{1}$ satisfies the conditions of Proposition 4.4. Observe that the map $j_{\mu}: L^{1}(\mu) \rightarrow \mathcal{S}_{\mathrm{BL}}$ is continuous, hence continuous for the weak topologies in $L^{1}(\mu)$ and $\mathcal{S}_{\mathrm{BL}}$ [4, Theorem VI.1.1]. The map

$$
\psi_{E}:[0, \tau] \rightarrow \mathcal{S}_{\mathrm{BL}}: t \mapsto P(t) j_{\mu}\left(\mathbb{1}_{E}\right)
$$


is norm continuous, hence continuous for the weak topology in $\mathcal{S}_{\mathrm{BL}}$. Thus its graph is closed in $[0, \tau] \times\left(\mathcal{S}_{\mathrm{BL}}\right)_{w}$. We conclude that $t \mapsto T(t) \mathbb{1}_{E}$ must have a closed graph in $[0, \tau] \times L_{w}^{1}$.

Now we will show that $C:=\left\{T(t) \mathbb{1}_{E}: 0 \leq t \leq \tau\right\}$ is compact in $L_{w}^{1}$. Note that $C=j_{\mu}^{-1}\left(\psi_{E}([0, \tau])\right)$. Thus $C$ is closed in $L_{w}^{1}$ by continuity of $j_{\mu}: L_{w}^{1} \rightarrow\left(\mathcal{S}_{\mathrm{BL}}\right)_{w}$ and compactness of $\psi_{E}([0, \tau])$ in $\left(\mathcal{S}_{\mathrm{BL}}\right)_{w}$. According to Theorem 4.5 it suffices to show that for any sequence of disjoint measurable subsets $A_{n}$ of $S$,

$$
\lim _{n \rightarrow \infty} \sup _{0 \leq t \leq \tau} \int_{A_{n}} T(t) \mathbb{1}_{E} d \mu=0 .
$$

We have $\mathbb{1}_{E} \leq \mathbb{1}$, thus $0 \leq T(t) \mathbb{1}_{E} \leq T(t) \mathbb{1}$ by positivity of $T(t)$. Therefore,

$$
\int_{A_{n}} T(t) \mathbb{1}_{E} d \mu \leq \int_{A_{n}} T(t) \mathbb{1} d \mu=P(t) \mu\left(A_{n}\right) .
$$

Condition (5) now implies (6). Now we can conclude that $C$ is weakly compact. Hence by Proposition $4.4, t \mapsto T(t) \mathbb{1}_{E}:[0, \tau] \rightarrow L_{w}^{1}$ is continuous.

Step 2. $(T(t))_{t \geq 0}$ is strongly continuous.

The proof of this step mimics that of [12, Theorem I.5.8] ('a weakly continuous semigroup in a Banach space is strongly continuous'). We will show that there is a norm dense subspace $D$ of $L^{1}(\mu)$, such that $t \mapsto T(t) f$ is norm continuous at zero for $f \in D$. Then $(T(t))_{t \geq 0}$ is strongly continuous on $L^{1}(\mu)$, because $\|T(t)\|=1$ for all $t$ (e.g. [12, Proposition I.5.3]).

By Step 1 and linearity, $t \mapsto T(t) f: \mathbb{R}_{+} \rightarrow L_{w}^{1}$ is continuous whenever $f$ is a step function. By separability of $S$ and Pettis' Theorem we conclude that for any step function $f, t \mapsto T(t) f$ is measurable in the sense of Bochner. It is integrable over $[0, \tau]$, because $\|T(t) f\|_{1} \leq\|f\|_{1}$. Thus we can define as Bochner integral in $L^{1}(\mu)$ :

$$
f_{r}:=\frac{1}{r} \int_{0}^{r} T(t) f d t, \quad 0<r \leq \tau .
$$

Because $t \mapsto T(t) f$ is weakly continuous, $f_{r} \rightarrow f$ weakly as $r \downarrow 0$. Thus

$$
D:=\operatorname{span}\left\{f_{r}: f \text { step function, } 0<r \leq \tau\right\}
$$

is weakly dense in $L^{1}(\mu)$. Because norm closure and weak closure agree on convex sets, $D$ is also a norm dense subspace of $L^{1}(\mu)$. Now, fix $r>0$ and let $0 \leq s \leq r$. Then for any step function $f$,

$$
\begin{aligned}
\left\|T(s) f_{r}-f_{r}\right\|_{1} & =\frac{1}{r}\left\|\int_{s}^{s+r} T(t) f d t-\int_{0}^{r} T(t) f d t\right\|_{1} \\
& =\frac{1}{r}\left\|\int_{r}^{s+r} T(t) f d t-\int_{0}^{s} T(t) f d t\right\|_{1} \leq\|f\|_{1} \frac{2 s}{r} .
\end{aligned}
$$

Thus $\left\|T(s) f_{r}-f_{r}\right\|_{1} \rightarrow 0$ as $s \downarrow 0$. By linearity $t \mapsto T(t) g$ is norm continuous at zero for every $g \in D$. Since $D$ is a norm dense subspace of $L^{1}(\mu),(T(t))_{t \geq 0}$ is strongly continuous on $L^{1}(\mu)$. 
Remark 4.7 (on the proof of Theorem 4.6) Let $\mu$ be as in the theorem. Then $\left\|P(t) j_{\mu}(f)-P(s) j_{\mu}(f)\right\|_{\mathrm{TV}}=\|T(t) f-T(s) f\|_{1}$ for every $t, s \in \mathbb{R}_{+}$and $f \in$ $L^{1}(\mu)$. This is what we use to prove (i) $\Rightarrow$ (ii). However it does not seem to be possible to prove (ii) $\Rightarrow$ (i) directly using this identity, since the continuity of $t \mapsto P(t) j_{\mu}(f)$ for general $f \in L^{1}(\mu)$ is not an easy consequence of the continuity of $t \mapsto P(t) \mu$. For this the 'detour' we take via (iii) seems to be necessary.

If $\mu \in \mathcal{M}^{+}(S)$ is an invariant measure of $(P(t))_{t \geq 0}$, i.e. $P(t) \mu=\mu$ for every $t \in \mathbb{R}_{+}$, then $(P(t))_{t \geq 0}$ leaves $j_{\mu}\left(L_{+}^{1}(\mu)\right)$ invariant by Corollary 4.3. Also, condition (ii) of Theorem 4.6 is satisfied, so the induced semigroup on $L^{1}(\mu)$ is strongly continuous.

Not every strongly stochastically continuous Markov semigroup which leaves $j_{\mu}\left(L_{+}^{1}(\mu)\right)$ invariant for some $\mu \in \mathcal{M}^{+}(S)$ satisfies one of the equivalent conditions of Theorem 4.6, as the following example will show. Let $m$ denote the Lebesgue measure on $\mathbb{R}^{n}$. The diffusion semigroup $\left(T_{d}(t)\right)_{t>0}$ on $L^{1}\left(\mathbb{R}^{n}\right)=L^{1}\left(\mathbb{R}^{n}, m\right)$ is given by

$$
T_{d}(t) f(x):=\int_{\mathbb{R}^{n}} h_{d}(x-y, t) f(y) d m(y), \quad \text { for } t>0,
$$

where the diffusion kernel $h_{d}$ is given by

$$
h_{d}(x, t)=(4 \pi d t)^{-n / 2} e^{-|x|^{2} / 4 d t} .
$$

Let $\mu \in \mathcal{M}\left(\mathbb{R}^{n}\right)$, then one can show that $x \mapsto g_{\mu}(x)=\int_{\mathbb{R}} h_{d}(x-y, t) f(y) d \mu(y)$ is in $L^{1}\left(\mathbb{R}^{n}\right)$, and hence defines a measure $g_{\mu} d m$. We can extend $T_{d}(t)$ to a map $P_{d}(t)$ : $\mathcal{M}\left(\mathbb{R}^{n}\right) \rightarrow \mathcal{M}\left(\mathbb{R}^{n}\right)$, by defining $P_{d}(t) \mu$ to be $g_{\mu} d m$. Then $P_{d}(t)$ is linear, leaves $\mathcal{M}^{+}\left(\mathbb{R}^{n}\right)$ invariant, and $\left\|P_{d}(t) \mu\right\|_{\mathrm{BL}}^{*} \leq\|\mu\|_{\mathrm{BL}}^{*}$ for all $\mu \in \mathcal{M}\left(\mathbb{R}^{n}\right)$, so $P_{d}(t)$ can be extended to a bounded linear operator $\left(\overline{P_{d}(t)}\right)_{t \geq 0}$ on $\mathbb{R}_{\mathrm{BL}}^{n}$. Moreover, $\left(\overline{P_{d}(t)}\right)_{t \geq 0}$ is strongly continuous on $\mathbb{R}_{\mathrm{BL}}^{n}$. Hence $\left(P_{d}(t)\right)_{t \geq 0}$ is a strongly stochastically continuous Markov semigroup by Lemma 3.5. Note that $P_{d}(t) \mu \ll m$ for every $\mu \in \mathcal{M}\left(\mathbb{R}^{n}\right)$ and $t>0$. Now let $f \in L^{1}\left(\mathbb{R}^{n}\right)$ such that $f>0$ almost everywhere, and set $\mu=$ $f d m+\delta_{0}$. Then $P_{d}(t)(\mu) \ll \mu$ for all $t \geq 0$, so $\left(P_{d}(t)\right)_{t \geq 0}$ leaves $L_{+}^{1}(\mu)$ invariant by Corollary 4.3. But $P_{d}(t) \mu \in L^{1}\left(\mathbb{R}^{n}\right)$ for all $t>0$, hence $t \mapsto P_{d}(t) \mu$ cannot be continuous from $\mathbb{R}_{+}$to $\mathcal{M}\left(\mathbb{R}^{n}\right)_{\mathrm{TV}}$, since $L^{1}\left(\mathbb{R}^{n}\right)$ is closed in $\mathcal{M}\left(\mathbb{R}^{n}\right)_{\mathrm{TV}}$ and $\mu \notin$ $L^{1}\left(\mathbb{R}^{n}\right)$, so condition (ii) of Theorem 4.6 is not satisfied.

\section{Strong continuity for total variation norm}

Let $(P(t))_{t \geq 0}$ be a strongly stochastically continuous Markov semigroup on $S$. It extends to a positive semigroup of bounded linear operators on $\mathcal{M}(S)_{\mathrm{TV}}$ as we have seen. Typically the latter is not strongly continuous. In this section we will give several characterisations of the closed invariant subspace of $\mathcal{M}(S)_{\mathrm{TV}}$ on which $(P(t))_{t \geq 0}$ is strongly continuous, i.e. the space

$$
\mathcal{M}(S)_{\mathrm{TV}}^{0}:=\left\{\mu \in \mathcal{M}(S): t \mapsto P(t) \mu \text { is continuous from } \mathbb{R}_{+} \text {to } \mathcal{M}(S)_{\mathrm{TV}}\right\}
$$


of $C^{0}$-vectors in $\mathcal{M}(S)$ for the $\|\cdot\|_{\mathrm{TV}}$-topology.

Our approach is based on that of Gulick et al. [15]. There the following situation is considered: A locally compact group $G$ acts as a group of homeomorphisms $\left(\Phi_{g}\right)_{g \in G}$ on a locally compact Hausdorff space $X$, sending $x \in X$ to $\Phi_{g}(x)$. This induces an action $(P(g))_{g \in G}$ on the Banach space of bounded Radon measures on $X, \mathcal{M}(X)$, endowed with total variation norm, given by $P(g) \mu(E):=\mu\left(\Phi_{g^{-1}} E\right)$. The subspace of $\mathcal{M}(X)$, consisting of measures $\mu$ such that $g \mapsto P(g) \mu$ is continuous from $G$ to $\mathcal{M}(X)$ is then identified using convolution of certain functions on $G$ with Radon measures on $X$, and this identification is used to provide several characterisations of this subspace (see also [21]).

Adopting this approach to our setting is not straightforward: Instead of a group $G$ as in [15], we consider a semigroup $\mathbb{R}_{+}$, which implies that actions need not be invertible. Also, in [15] an action of the group on the underlying space $X$ is considered, which induces an action on $\mathcal{M}(X)$. While we look, more generally, at actions of $\mathbb{R}_{+}$on $\mathcal{M}(S)$ directly, that contain those coming from an underlying action on $S$ by Proposition 3.6, which need not be continuous, only measurable. Furthermore, in [15] $X$ must be locally compact, since measures on $X$ are defined there by constructing certain functionals on $C_{0}(X)$; in our setting $S$ needs to be a separable complete metric space, but not necessarily locally compact. We can however overcome these difficulties by using the Banach space $\mathcal{S}_{\mathrm{BL}}$ and the theory of integrating functions with values in $\mathcal{S}_{\mathrm{BL}}^{+}=\mathcal{M}^{+}(S)$ as developed in Sect. 2.3 and prove analogous characterisations of $\mathcal{M}(S)_{\mathrm{TV}}^{0}$ as those in [15] and [21].

These characterisations will help in identifying when the restriction of $(P(t))_{t \geq 0}$ to invariant $L^{1}$-spaces is strongly continuous.

Let $A$ be a Banach algebra with multiplication $*$. A net $\left(e_{\alpha}\right)$ in $A$ is an approximate identity of $A$, if $\lim _{\alpha} e_{\alpha} * f=f$ and $\lim _{\alpha} f * e_{\alpha}=f$ for all $f \in A$. It is a bounded approximate identity if the net is bounded. A Banach space $M$ is a Banach module over $A$ if there exists a bilinear map $\star: A \times M \rightarrow M$ having the following properties:

(BM1) $(f * g) \star m=f \star(g \star m)$ for all $f, g \in A, m \in M$

(BM2) $\|f \star m\|_{M} \leq\|f\|_{A}\|m\|_{M}$ for all $f \in A, m \in M$.

Proposition 5.1 ([15, Corollary 2.3]) Let A be a Banach algebra with bounded approximate identity $\left(e_{\alpha}\right)$. If $M$ is a Banach module over $A$, then $A \star M:=\{a \star m: a \in$ $A, m \in M\}$ is a closed subspace of $K$. In particular, for $m \in M, m \in A \star M$ if and only if $\lim _{\alpha} e_{\alpha} \star m=m$.

The latter characterisation of elements in $A \star M$ shows that $A \star M$ is indeed a vector subspace of $M$.

Proposition 5.2 The Banach space $L^{1}\left(\mathbb{R}_{+}\right)$is a commutative Banach algebra with multiplication defined by convolution:

$$
f * g(t):=\int_{0}^{t} f(t-s) g(s) d s,
$$

with bounded approximate identity $\left(e_{n}\right)$ given by $e_{n}=n \mathbb{1}_{\left[0, \frac{1}{n}\right]}$. 
The proof is straightforward, observing that $L^{1}\left(\mathbb{R}_{+}\right)$is canonically contained as closed subspace in the commutative Banach algebra $L^{1}(\mathbb{R})$ with convolution.

For a strongly stochastically continuous Markov semigroup $(P(t))_{t \geq 0}, t \mapsto$ $P(t) \mu, \mathbb{R}_{+} \rightarrow \mathcal{S}_{\mathrm{BL}}$ is continuous for each $\mu \in \mathcal{M}(S)$ by Lemma 3.5 (though $P(t)$ : $\mathcal{M}(S)_{\mathrm{BL}} \rightarrow \mathcal{M}(S)_{\mathrm{BL}}$ need not be continuous) and

$$
\|P(t) \mu\|_{\mathrm{BL}}^{*} \leq\|P(t) \mu\|_{\mathrm{TV}} \leq\|\mu\|_{\mathrm{TV}} .
$$

Thus $P(\cdot) \mu \in C_{b}\left(\mathbb{R}_{+}, \mathcal{S}_{\mathrm{BL}}\right)$ and we can define for $f \in L^{1}\left(\mathbb{R}_{+}\right)$and $\mu \in \mathcal{M}(S)$

$$
f *_{P} \mu:=\int_{\mathbb{R}_{+}} f(s) P(s) \mu d s
$$

as Bochner integral in $\mathcal{S}_{\mathrm{BL}}$. Clearly $(f, \mu) \mapsto f *_{P} \mu$ is a bilinear map from $L^{1}\left(\mathbb{R}_{+}\right) \times \mathcal{M}(S)$ to $\mathcal{S}_{\mathrm{BL}}$. Because $\mathcal{S}_{\mathrm{BL}}^{+}$is closed and convex in $\mathcal{S}_{\mathrm{BL}}, f *_{P} \mu \in$ $\mathcal{S}_{\mathrm{BL}}^{+}=\mathcal{M}^{+}(S)$, when $f \in L_{+}^{1}\left(\mathbb{R}_{+}\right)$and $\mu \in \mathcal{M}^{+}(S)$. By writing $f \in L^{1}\left(\mathbb{R}_{+}\right)$and $\mu \in \mathcal{M}(S)$ as difference of positive and negative parts $f^{ \pm}$and $\mu^{ \pm}$respectively, it follows that

$$
f *_{P} \mu=f^{+} *_{P} \mu^{+}-f^{-} *_{P} \mu^{+}-f^{+} *_{P} \mu^{-}+f^{-} *_{P} \mu^{-} .
$$

So $(f, \mu) \mapsto f *_{P} \mu$ is a bilinear map from $L^{1}\left(\mathbb{R}_{+}\right) \times \mathcal{M}(S)$ into $\mathcal{M}(S)$.

The right translation semigroup $\left(R_{+}(t)\right)_{t \geq 0}$ on $L^{1}\left(\mathbb{R}_{+}\right)$is given by:

$$
R_{+}(t) f(s):= \begin{cases}f(s-t), & \text { if } s \geq t \\ 0, & \text { if } 0 \leq s \leq t .\end{cases}
$$

It is a strongly continuous positive semigroup on $L^{1}\left(\mathbb{R}_{+}\right)$.

Proposition 5.3 The following holds for all $f \in L^{1}\left(\mathbb{R}_{+}\right), \mu \in \mathcal{M}(S)$ :

(i) Let $P(t)$ be regular. Then

$$
P(t)\left(f *_{P} \mu\right)=f *_{P}(P(t) \mu)=\left(R_{+}(t) f\right) *_{P} \mu
$$

(ii) $f *_{P} \mu(E)=\int_{\mathbb{R}_{+}} f(t) P(t) \mu(E) d t$ for all Borel sets $E$ in $S$.

Proof Since $P(t)$ is regular, there exists an $U(t): \mathrm{BM}(S) \rightarrow \operatorname{BM}(S)$ such that $\langle P(t) \mu, h\rangle=\langle\mu, U(t) h\rangle$ for all $\mu \in \mathcal{M}(S), h \in \mathrm{BM}(S)$. It suffices to prove (i) for $f \in L_{+}^{1}\left(\mathbb{R}_{+}\right)$and $\mu \in \mathcal{M}^{+}(S)$. The general statement follows then from (7). Note that $f d s$ defines a measure in $\mathcal{M}^{+}\left(\mathbb{R}_{+}\right)$. Let $h \in \mathrm{BM}(S)$.

$$
\begin{aligned}
\left\langle P(t)\left(f *_{P} \mu\right), h\right\rangle & =\left\langle f *_{P} \mu, U(t) h\right\rangle \\
& =\int_{S} U(t) h d\left[\int_{\mathbb{R}_{+}} f(s) P(s) \mu d s\right] .
\end{aligned}
$$

By Proposition 2.6 we get

$$
\int_{S} U(t) h d\left[\int_{\mathbb{R}_{+}} f(s) P(s) \mu d s\right]=\int_{\mathbb{R}_{+}}\langle P(s) \mu, U(t) h\rangle f(s) d s
$$




$$
=\int_{\mathbb{R}_{+}}\langle P(t+s) \mu, h\rangle f(s) d s .
$$

Again applying Proposition 2.6 yields that (8) equals

$$
\int_{S} h d\left[\int_{\mathbb{R}_{+}} f(s) P(t+s) \mu d s\right]=\left\langle f *_{P}(P(t) \mu), h\right\rangle .
$$

Hence $P(t)\left(f *_{P} \mu\right)=f *_{P}(P(t) \mu)$.

The map $s \mapsto f(s) P(t+s) \mu$ is Bochner integrable from $\mathbb{R}_{+}$to $\mathcal{S}_{\mathrm{BL}}$. Using the fact that Lebesgue measure on $\mathbb{R}$ is invariant under translation,

$$
\begin{aligned}
\int_{\mathbb{R}_{+}} f(s) P(t+s) \mu d s & =\int_{\mathbb{R}_{+}}\left(R_{+}(t) f\right)(s) P(s) \mu d s \\
& =\left(R_{+}(t) f\right) * P \mu .
\end{aligned}
$$

The statement in (ii) follows from (7) and Corollary 2.7.

From this point on we will implicitly assume that $(P(t))_{t \geq 0}$ is regular.

Proposition 5.4 Let $f, g \in L^{1}\left(\mathbb{R}_{+}\right)$and $\mu \in \mathcal{M}(S)$, then

(i) $(f * g) *_{P} \mu=f *_{P}\left(g *_{P} \mu\right)$

(ii) $\left\|f *_{P} \mu\right\|_{\mathrm{TV}} \leq\|f\|_{1}\|\mu\|_{\mathrm{TV}}$.

Consequently, $\mathcal{M}(S)_{\mathrm{TV}}$ is a Banach module over $L^{1}\left(\mathbb{R}_{+}\right)$.

Proof We first prove (i). We use Fubini's Theorem for Bochner integration [16, Theorem 3.7.13] and Proposition 5.3:

$$
\begin{aligned}
(f * g) *_{P} \mu & =\int_{\mathbb{R}_{+}}\left\{\int_{\mathbb{R}_{+}} f(s) g(t-s) d s\right\} P(t) \mu d t \\
& =\int_{\mathbb{R}_{+}} f(s)\left\{\int_{\mathbb{R}_{+}} g(t-s) P(t) \mu d t\right\} d s \\
& =\int_{\mathbb{R}_{+}} f(s)\left(R_{+}(s) g\right) * P \mu d s \\
& =\int_{\mathbb{R}_{+}} f(s) P(s)(g * P \mu) d s \\
& =f *_{P}(g * P \mu) .
\end{aligned}
$$

For $f \in L_{+}^{1}\left(\mathbb{R}_{+}\right)$and $v \in \mathcal{M}^{+}(S), f *_{P} v \in \mathcal{M}^{+}(S)$ and

$$
\begin{aligned}
\left\|f *_{P} v\right\|_{\mathrm{TV}}=\left\|f *_{P} v\right\|_{\mathrm{BL}}^{*} & \leq \int_{\mathbb{R}_{+}}\|f(t) P(t) v\|_{\mathrm{BL}}^{*} d t \\
& =\int_{\mathbb{R}_{+}} f(t)\|P(t) v\|_{\mathrm{TV}} d t=\|v\|_{\mathrm{TV}}\|f\|_{1}
\end{aligned}
$$


by using property (MO2). For general $f \in L^{1}\left(\mathbb{R}_{+}\right)$and $\mu \in \mathcal{M}(S)$ we then obtain

$$
\begin{aligned}
\left\|f *_{P} \mu\right\|_{\mathrm{TV}} & \leq\left(\left\|f^{+}\right\|_{1}+\left\|f^{-}\right\|_{1}\right)\left\|\mu^{+}\right\|_{\mathrm{TV}}+\left(\left\|f^{+}\right\|_{1}+\left\|f^{-}\right\|_{1}\right) *\left\|\mu^{-}\right\|_{\mathrm{TV}} \\
& =\|f\|_{1}\left(\left\|\mu^{+}\right\|_{\mathrm{TV}}+\left\|\mu^{-}\right\|_{\mathrm{TV}}\right)=\|f\|_{1}\|\mu\|_{\mathrm{TV}},
\end{aligned}
$$

by using (7) and the fact that $\mathcal{M}(S)$ and $L^{1}\left(\mathbb{R}_{+}\right)$are $L$-spaces.

So $\mathcal{M}(S)_{\mathrm{TV}}$ is a Banach module over $L^{1}\left(\mathbb{R}_{+}\right)$.

Put $L^{1}\left(\mathbb{R}_{+}\right) *_{P} \mathcal{M}(S):=\left\{f *_{P} \mu: f \in L^{1}\left(\mathbb{R}_{+}\right), \mu \in \mathcal{M}(S)\right\}$.

Then we have, by Proposition 5.2, Proposition 5.4 and Proposition 5.1, the following result:

Corollary $5.5 L^{1}\left(\mathbb{R}_{+}\right) *_{P} \mathcal{M}(S)$ is a non-trivial closed subspace of $\mathcal{M}(S)_{\mathrm{TV}}$.

This closed subspace equals the subspace of strong continuity of $P(t)$ with respect to $\|\cdot\|_{\mathrm{TV}}$ :

Theorem 5.6 For $\mu \in \mathcal{M}(S)$ the following are equivalent:

(i) $\mu \in \mathcal{M}(S)_{\mathrm{TV}}^{0}$, i.e. $t \mapsto P(t) \mu: \mathbb{R}_{+} \rightarrow \mathcal{M}(S)_{\mathrm{TV}}$ is continuous

(ii) $\mu \in L^{1}\left(\mathbb{R}_{+}\right) *_{P} \mathcal{M}(S)$

(iii) if $E$ is a Borel set in $S$ such that $P(t) \mu(E)=0$ for almost every $t \in[0, \infty)$, then $\mu(E)=0$

(iv) there exists $v \in \mathcal{M}^{+}(S)_{\mathrm{TV}}^{0}$ such that $j_{v}\left(L^{1}(v)\right)$ is $(P(t))_{t \geq 0}$-invariant and $\mu \in$ $j_{v}\left(L^{1}(v)\right)$.

Proof (i) $\Rightarrow$ (ii): Let $\mu \in \mathcal{M}(S)_{\mathrm{TV}}^{0}$. By Proposition 5.1 it is sufficient to show that $e_{n} * \mu \rightarrow \mu$. Let $\epsilon>0$. Since $t \mapsto P(t) \mu: \mathbb{R}_{+} \rightarrow \mathcal{M}(S)_{\mathrm{TV}}$ is continuous, there exists an $N \in \mathbb{N}$, such that $\|P(t) \mu-\mu\|_{\mathrm{TV}} \leq \epsilon$ for all $t \in\left[0, \frac{1}{N}\right]$. For $n \in \mathbb{N}$

$$
e_{n} * \mu-\mu=n \int_{0}^{\frac{1}{n}} P(t) \mu-\mu d t
$$

is defined as Bochner integral in $\mathcal{S}_{\mathrm{BL}}$.

By continuity, $t \mapsto P(t) \mu-\mu:\left[0, \frac{1}{n}\right] \rightarrow \mathcal{M}(S)_{\mathrm{TV}}$ is strongly measurable and bounded, hence Bochner integrable, so we can also view the integral $n \int_{0}^{\frac{1}{n}} P(t) \mu-$ $\mu d s$ as a Bochner integral in $\mathcal{M}(S)_{\mathrm{TV}}$. Since $\mathcal{M}(S)_{\mathrm{TV}}$ embeds continuously in $\mathcal{S}_{\mathrm{BL}}$, the two integrals are the same.

Moreover,

$$
\left\|e_{n} * P \mu-\mu\right\|_{\mathrm{TV}} \leq n \int_{0}^{\frac{1}{n}}\|P(t) \mu-\mu\|_{\mathrm{TV}} d t \leq \epsilon,
$$

for all $n \geq N$.

(ii) $\Rightarrow$ (i): Let $\mu=f *_{P} v \in L^{1}\left(\mathbb{R}_{+}\right) *_{P} \mathcal{M}(S)$. Let $t, s \geq 0$. According to Proposition 5.3 and Proposition 5.4,

$$
\|P(t) \mu-\mu\|_{\mathrm{TV}}=\left\|\left(R_{+}(t) f\right) *_{P} v-f *_{P} v\right\|_{\mathrm{TV}} \leq\left\|R_{+}(t) f-f\right\|_{1}\|v\|_{\mathrm{TV}} .
$$


Since $\left(R_{+}(t)\right)_{t \geq 0}$ is strongly continuous on $L^{1}\left(\mathbb{R}_{+}\right),\left\|R_{+}(t) f-f\right\|_{1} \rightarrow 0$ as $t \downarrow 0$ and thus $\|P(t) \mu-\mu\|_{\mathrm{TV}} \rightarrow 0$. So $\mu \in \mathcal{M}(S)_{\mathrm{TV}}^{0}$.

Thus from now on we can identify $\mathcal{M}(S)_{\mathrm{TV}}^{0}$ with $L^{1}\left(\mathbb{R}_{+}\right) *_{P} \mathcal{M}(S)$.

(i) $\Rightarrow$ (iii): Let $\mu \in \mathcal{M}(S)_{\mathrm{TV}}^{0}$ and let $E$ be a Borel set in $S$. Then $t \mapsto P(t) \mu(E)$ is continuous, hence if $P(t) \mu(E)=0$ for almost every $t \in[0, \infty)$, then $\mu(E)=0$.

(iii) $\Rightarrow$ (iv) Let $f \in L^{1}\left(\mathbb{R}_{+}\right)$, such that $f(t)>0$ for almost every $t \in[0, \infty)$. Define $v=f *_{P}|\mu|$. Suppose $v(E)=0$ for a Borel set $E$ in $S$, then $P(t)|\mu|(E)=0$ for almost every $t \in[0, \infty)$. By positivity of $P(t),|P(t) \mu|(E) \leq P(t)|\mu|(E)=0$ for almost every $t \in[0, \infty)$, hence $\mu(E)=0$ and $\mu \ll v$. Furthermore, by Proposition 5.3,

$$
P(t) v(E)=\int_{\mathbb{R}_{+}} f(s) P(t+s)|\mu|(E) d s=0,
$$

hence $P(t) v \ll v$. According to Corollary 4.3, $(P(t))_{t \geq 0}$ leaves $j_{v}\left(L^{1}(v)\right)$ invariant, and $\mu \in j_{v}\left(L^{1}(v)\right)$.

(iv) $\Rightarrow$ (i): Since $v \in \mathcal{M}(S)_{\mathrm{TV}}^{0}, t \mapsto P(t) v: \mathbb{R}_{+} \rightarrow \mathcal{M}(S)_{\mathrm{TV}}$ is continuous. Then Theorem 4.6 implies that the semigroup $(T(t))_{t \geq 0}$ in $L^{1}(v)$ induced by $(P(t))_{t \geq 0}$ is strongly continuous. By assumption there is an $f \in L^{1}(v)$ such that $j_{v}(f)=\mu$. Then

$$
\|P(t) \mu-\mu\|_{\mathrm{TV}}=\|T(t) f-f\|_{1} \rightarrow 0,
$$

as $t \downarrow 0$.

The aim of the following theorem is to give some apparently weaker conditions than those in Theorem 5.6, which turn out to be equivalent. These may be useful for showing that a particular measure $\mu$ is in $\mathcal{M}(S)_{\mathrm{TV}}^{0}$.

Theorem 5.7 Let $\mu \in \mathcal{M}(S)$. Then the following are equivalent:

(i) $\mu \in \mathcal{M}(S)_{\mathrm{TV}}^{0}$

(ii) for all compact $K$ in $S, t \mapsto P(t) \mu(K)$ is continuous

(iii) if $K$ in $S$ compact and $P(t) \mu(K)=0$ for almost every $t \in[0, \infty)$, then $\mu(K)=0$

(iv) there is a $v \in \mathcal{M}(S)_{\mathrm{TV}}^{0}$ such that $\mu \ll v$.

Proof (i) $\Rightarrow$ (ii): Since $\mu \in \mathcal{M}(S)_{\mathrm{TV}}^{0}, t \mapsto P(t) \mu(E)$ is continuous for all Borel sets $E$ in $S$.

(ii) $\Rightarrow$ (iii): Let $K$ in $S$ be compact, such that $P(t) \mu(K)=0$ for almost every $t \in[0, \infty)$. Then, by continuity of $t \mapsto P(t) \mu(K), \mu(K)=0$.

(iii) $\Rightarrow$ (iv): Let $f \in L^{1}\left(\mathbb{R}_{+}\right)$, such that $f(t)>0$ for almost every $t \in[0, \infty)$. Define $v:=f * P|\mu|$. Let $K$ in $S$ be compact, such that $v(K)=0$, then $P(t)|\mu|(K)=0$ for almost every $t \in[0, \infty)$. By positivity of $P(t),|P(t) \mu|(K) \leq P(t)|\mu|(K)=0$ for almost every $t \in[0, \infty)$, hence $\mu(K)=0$. Thus $\mu \ll v$ by Lemma 2.1 .

(iv) $\Rightarrow$ (i): Let $f \in L^{1}\left(\mathbb{R}_{+}\right)$, such that $f(t)>0$ for almost every $t \in[0, \infty)$. Define $\rho:=f *_{P}|\nu| \in \mathcal{M}^{+}(S) \cap L^{1}\left(\mathbb{R}_{+}\right) *_{P} \mathcal{M}(S)$. Now, let $E$ be a Borel set in $S$ such that $\rho(E)=0$. Then $P(t)|\nu|(E)=0$ for almost every $t \in[0, \infty)$. By positivity of $P(t)$,

$$
|P(t) v(E)| \leq P(t)|v|(E)=0,
$$


for almost every $t \in[0, \infty)$. Since $v \in \mathcal{M}(S)_{\mathrm{TV}}^{0}, t \mapsto P(t) v(E)$ is continuous, so $v(E)=0$. So $\mu \ll v \ll \rho$.

Also, by Proposition 5.3, we have for every $s \geq 0$

$$
P(s) \rho(E)=\int_{\mathbb{R}_{+}} f(t) P(t+s)|v|(E) d t=0,
$$

since $P(t)|\nu|(E)=0$ for every $t \geq 0$. So $P(t) \rho \ll \rho$ for all $t \geq 0$, and $\mu \ll \rho$. By Corollary $4.3(P(t))_{t \geq 0}$ leaves $j_{\rho}\left(L^{1}(\rho)\right)$ invariant, and $\mu \in j_{\rho}\left(L^{1}(\rho)\right)$. Now we can apply Theorem 5.6.

Corollary 5.8 Let $\mu \in \mathcal{M}^{+}(S)$. If there is a $\tau>0$ such that $\mu \ll P(t) \mu$ for all $t \in[0, \tau]$, then $\mu \in \mathcal{M}(S)_{\mathrm{TV}}^{0}$.

Proof Let $E \subset S$ be measurable such that $P(t) \mu(E)=0$ for almost every $t \in[0, \infty)$. Then there is a $t \in[0, \tau]$ such that $P(t) \mu(E)=0$, and then $\mu(E)=0$, since $\mu \ll$ $P(t) \mu$. Hence $\mu \in \mathcal{M}(S)_{\mathrm{TV}}^{0}$ by Theorem 5.6.

If $\mu \in \mathcal{M}^{+}(S)$ is an invariant measure of $(P(t))_{t \geq 0}$, then $\mu \in \mathcal{M}(S)_{\mathrm{TV}}^{0}$, since $t \mapsto P(t) \mu=\mu$ is continuous from $\mathbb{R}_{+}$to $\mathcal{M}(S)_{\mathrm{TV}}$. It would be interesting to be able to characterise the invariant measures among those in $\mathcal{M}(S)_{\mathrm{TV}}^{0}$.

An important consequence of the characterisations in Theorem 5.6 is:

Proposition 5.9 $\mathcal{M}(S)_{\mathrm{TV}}^{0}$ is dense in $\mathcal{M}(S)_{\mathrm{BL}}$, hence in $\mathcal{S}_{\mathrm{BL}}$.

Proof Let $\mu \in \mathcal{M}(S)$ and $\epsilon>0$. Then there is a $\tau>0$ such that $\|P(t) \mu-\mu\|_{\mathrm{BL}}^{*}<\epsilon$ for all $t \in[0, \tau]$. By Theorem $5.6 e_{n} *_{P} \mu \in \mathcal{M}(S)_{\mathrm{TV}}^{0}$.

$$
\begin{aligned}
\left\|e_{n} *_{P} \mu-\mu\right\|_{\mathrm{BL}}^{*} & =n\left\|\int_{0}^{\frac{1}{n}} P(t) \mu-\mu d t\right\|_{\mathrm{BL}}^{*} \\
& \leq n \int_{0}^{\frac{1}{n}}\|P(t) \mu-\mu\|_{\mathrm{BL}}^{*} d t<\epsilon,
\end{aligned}
$$

for all $t \in[0, \tau]$. So $\left\|e_{n} *_{P} \mu-\mu\right\|_{\mathrm{BL}}^{*} \rightarrow 0$, and $\mathcal{M}(S)_{\mathrm{TV}}^{0}$ is dense in $\mathcal{M}(S)_{\mathrm{BL}}$.

However, whenever the Markov semigroup arises from a non-trivial underlying semigroup of measurable maps on $S, \mathcal{M}(S)_{\mathrm{TV}}^{0}$ cannot be too large:

Proposition 5.10 Let $\left(\Phi_{t}\right)_{t \geq 0}$ be a strongly continuous semigroup of measurable maps on $S$, and let $(P(t))_{t \geq 0}$ be the associated strongly stochastically continuous Markov semigroup. Then $\mathcal{M}(S)_{\mathrm{TV}}^{0}=\mathcal{M}(S)$ if and only if $\Phi_{t}=\operatorname{Id}$ for every $t \in \mathbb{R}_{+}$.

Proof Suppose $\Phi_{t}=$ Id for every $t \in \mathbb{R}_{+}$. Then $P(t) \mu=\mu$ for every $t \in \mathbb{R}_{+}$and $\mu \in \mathcal{M}(S)$, hence $\mathcal{M}(S)_{\mathrm{TV}}^{0}=\mathcal{M}(S)$. Suppose $\mathcal{M}(S)=\mathcal{M}(S)_{\mathrm{TV}}^{0}$, and let $x \in S$. Then

$$
\left\|\delta_{\Phi(t) x}-\delta_{x}\right\|_{\mathrm{TV}}=\left\|P(t) \delta_{x}-\delta_{x}\right\|_{\mathrm{TV}} \downarrow 0,
$$


as $t \downarrow 0$. Hence there is a $\tau>0$ such that $\delta_{\Phi_{t}(x)}=\delta_{x}$ for all $t \in[0, \tau)$, and then by the semigroup law $\delta_{\Phi_{t}(x)}=\delta_{x}$ for all $t \in \mathbb{R}_{+}$, so $\Phi_{t}(x)=x$ for all $t \in \mathbb{R}_{+}$.

However, there do exist non-trivial strongly stochastically continuous Markov semigroups $(P(t))_{t \geq 0}$ such that $\mathcal{M}(S)_{\mathrm{TV}}^{0}=\mathcal{M}(S)$; in [29, Sect. 5] a $C_{0}$-semigroup on $\mathcal{M}(\Omega)_{\mathrm{TV}}$, with $(\Omega, \Sigma)$ a general measurable space, is constructed, which under certain conditions is a Markov semigroup.

Remark 5.11 One might also consider semigroups $(P(t))_{t \geq 0}$ on $\mathcal{M}^{+}(S)$ for which the Markov operators $P(t)$ satisfy a more general condition than (MO2):

$$
\|P(t) \mu\|_{T V} \leq M e^{\lambda t}\|\mu\|_{\mathrm{TV}},
$$

for certain $M \geq 1$ and $\lambda \geq 0$. In this case we can still achieve results similar to Theorems 5.6 and 5.7 , using a weighted $L^{1}$-space instead of $L^{1}\left(\mathbb{R}_{+}\right)$

$$
L_{\lambda, M}^{1}\left(\mathbb{R}_{+}\right):=\left\{f \in L^{1}\left(\mathbb{R}_{+}\right): t \mapsto e^{\lambda t}|f(t)| \in L^{1}\left(\mathbb{R}_{+}\right)\right\}
$$

with norm $\|f\|_{\lambda, M}:=\int_{\mathbb{R}_{+}} M e^{\lambda t}|f(t)| d t$.

\section{Decomposition of the space of measures}

\subsection{Absolute continuous and singular measures}

For $\mu \in \mathcal{M}(\mathbb{R})$, define $\mu_{t}(E):=\mu(E-t), t \in \mathbb{R}$. It is a classical result by Plessner [26] that $\left\|\mu_{t}-\mu\right\|_{\mathrm{TV}} \rightarrow 0$ as $t \rightarrow 0$ if and only if $\mu$ is absolutely continuous with respect to the Lebesgue measure $m$. Then the Lebesgue-Radon-Nikodym Decomposition Theorem implies that every $\mu$ in $\mathcal{M}(\mathbb{R})$ can be uniquely decomposed into $\mu_{a}+\mu_{s}$, where $\mu_{a} \in L^{1}(\mathbb{R}, m)$, and $\mu_{s}$ is singular with respect to $m$.

We can translate this to our setting: let $\Phi_{t}(x)=x+t$, then $\left(\Phi_{t}\right)_{t \in \mathbb{R}}$ defines a strongly continuous group of continuous mappings $\Phi_{t}: \mathbb{R} \rightarrow \mathbb{R}$. This defines a strongly stochastically continuous Markov group $(P(t))_{t \in \mathbb{R}}$, by $P(t) \mu=\mu \circ \Phi_{t}^{-1}$, by Proposition 3.6. Note that we only formulated Proposition 3.6 for semigroups, but it can easily adapted for groups. Plessner's result implies that the subspace of strong continuity $\mathcal{M}(\mathbb{R})_{\mathrm{TV}}^{0}$ equals $L^{1}(\mathbb{R})$, and every $\mu \in \mathcal{M}(\mathbb{R})$ can be uniquely decomposed into $\mu_{a}+\mu_{s}$, where $\mu_{a} \in \mathcal{M}(\mathbb{R})_{\mathrm{TV}}^{0}$ and $\mu_{s}$ is singular with respect to every $v \in \mathcal{M}(\mathbb{R})_{\mathrm{TV}}^{0}$. We will generalise this decomposition in our setting.

As in the previous section we assume $(P(t))_{t \geq 0}$ is a strongly stochastically continuous regular Markov semigroup on $S$.

Proposition 6.1 $\mathcal{M}(S)_{\mathrm{TV}}^{0}$ is a projection band in $\mathcal{M}(S)_{\mathrm{TV}}$.

Proof We first show that $\mathcal{M}(S)_{\mathrm{TV}}^{0}$ is an ideal. Let $\mu, v \in \mathcal{M}(S)$ such that $0 \leq$ $|\mu| \leq|\nu|$ and $\nu \in \mathcal{M}(S)_{\mathrm{TV}}^{0}$. Then $|\nu| \in \mathcal{M}(S)_{\mathrm{TV}}^{0}$ by Theorem 5.7. Since $\mu \ll|v|$, $\mu \in \mathcal{M}(S)_{\mathrm{TV}}^{0}$, again by Theorem 5.7. Hence $\mathcal{M}(S)_{\mathrm{TV}}^{0}$ is a closed ideal in $\mathcal{M}(S)_{\mathrm{TV}}$, hence a projection band by Theorem 2.2. 
So we can write

$$
\mathcal{M}(S)=\mathcal{M}(S)_{\mathrm{TV}}^{0} \oplus\left(\mathcal{M}(S)_{\mathrm{TV}}^{0}\right)^{\perp},
$$

by Theorem [22, Theorem 1.2.9].

We will show that $\left(\mathcal{M}(S)_{T V}^{0}\right)^{\perp}=\mathcal{M}(S)_{\mathrm{TV}}^{s}$, where

$\mathcal{M}(S)_{\mathrm{TV}}^{S}:=\left\{\mu \in \mathcal{M}(S): \mu^{+} \perp P(t) \mu^{+}, \mu^{-} \perp P(t) \mu^{-}\right.$for almost every $\left.t \geq 0\right\}$.

Our approach is based on that by Liu and Van Rooij [21].

Proposition 6.2 Let $\mu \in \mathcal{M}(S)$. Then the following are equivalent:

(i) $\mu \in \mathcal{M}(S)_{\mathrm{TV}}^{s}$

(ii) $\mu \perp v$ for every $v \in \mathcal{M}(S)_{\mathrm{TV}}^{0}$

(iii) for all $v \in \mathcal{M}(S), \mu \perp P(t) v$ for almost every $t \in[0, \infty)$.

Proof (i) $\Rightarrow$ (ii): Let $v \in \mathcal{M}(S)_{\mathrm{TV}}^{0}$, then $|v| \in \mathcal{M}(S)_{\mathrm{TV}}^{0}$ by Theorem 5.7. By the Lebesgue-Radon-Nikodym Theorem, there are unique $\mu_{a}^{+}, \mu_{s}^{+} \in \mathcal{M}(S)^{+}$, such that $\mu^{+}=\mu_{a}^{+}+\mu_{s}^{+}, \mu_{a}^{+} \ll|v|$ and $\mu_{s}^{+} \perp|v|$. Then $\mu_{a}^{+} \in \mathcal{M}(S)_{\mathrm{TV}}^{0}$ by Theorem 5.7. By assumption, $\mu^{+} \perp P(t) \mu^{+}$for almost every $t \in[0, \infty)$. Suppose $\mu^{+} \perp P(t) \mu^{+}$, then there is a Borel set $U$, such that $\mu^{+}(E)=\mu^{+}(E \cap U)$ and $P(t) \mu^{+}(U)=0$ for all Borel sets $E$. So

$$
0 \leq \mu_{a}^{+}(E \backslash U) \leq \mu^{+}(E \backslash U)=0,
$$

hence $\mu_{a}^{+}(E)=\mu_{a}^{+}(E \cap U)$ for all Borel sets $E$, and

$$
0 \leq P(t) \mu_{a}^{+}(U) \leq P(t) \mu^{+}(U)=0,
$$

so $P(t) \mu_{a}^{+} \perp \mu_{a}^{+}$.

Hence $\mu_{a}^{+} \perp P(t) \mu_{a}^{+}$for almost every $t \in[0, \infty) .\left\{\mu_{a}^{+}\right\}^{\perp}$ is a band in $\mathcal{M}(S)_{\mathrm{TV}}$, hence closed. Since $t \mapsto P(t) \mu_{a}^{+}: \mathbb{R}_{+} \rightarrow \mathcal{M}(S)_{\mathrm{TV}}$ is continuous, $\mu_{a}^{+} \in\left\{\mu_{a}^{+}\right\}^{\perp}$, thus $\mu_{a}^{+}=0$. This implies that $\mu^{+}=\mu_{s}^{+}$, so $\mu^{+} \perp|v|$, and therefore $\mu^{+} \perp v$.

In a similar way we can prove that $\mu^{-} \perp v$, hence $\mu \perp v$.

(ii) $\Rightarrow$ (iii): Let $v \in \mathcal{M}(S)$ and define $\rho:=f *_{P}|\nu| \in L^{1}\left(\mathbb{R}_{+}\right) *_{P} \mathcal{M}(S)$, where $f \in L^{1}\left(\mathbb{R}_{+}\right)$, such that $f(t)>0$ for almost every $t \in[0, \infty)$. Then $\rho \in \mathcal{M}(S)_{\mathrm{TV}}^{0}$ by Theorem 5.6. By (ii) $\mu \perp \rho$, hence there is a Borel set $U \subset S$, such that $\mu(E)=$ $\mu(E \cap U)$ and $\rho(U)=0$ for all Borel sets $E$ in $S$. By Corollary 2.7 $P(t)|v|(U)=0$ for almost every $t \in[0, \infty)$. Then positivity of $(P(t))_{t \geq 0}$ implies that for almost every $t \in[0, \infty),|P(t) \nu|(U)=0$, hence $|P(t) \nu| \perp \mu$. So $P(t) \nu \perp \mu$ for almost every $t \in[0, \infty)$.

(iii) $\Rightarrow$ (i): By assumption, $\mu \perp P(t) \mu^{+}$and $\mu \perp P(t) \mu^{-}$for almost every $t \in[0, \infty)$. Hence $|\mu| \perp P(t) \mu^{+}$and $|\mu| \perp P(t) \mu^{-}$, so $\mu^{+} \perp P(t) \mu^{+}$and $\mu^{-} \perp$ $P(t) \mu^{-}$for almost every $t \in[0, \infty)$.

Corollary 6.3 $\mathcal{M}(S)_{\mathrm{TV}}^{s}=\left(\mathcal{M}(S)_{\mathrm{TV}}^{0}\right)^{\perp}$.

This implies that $\mathcal{M}(S)_{\mathrm{TV}}^{s}$ is a projection band by [22, Proposition 1.2.7]. 
As in [21] we call $\mu \in \mathcal{M}(S)$ absolutely continuous with respect to $(P(t))_{t \geq 0}$ if $\mu \in \mathcal{M}(S)_{\mathrm{TV}}^{0}$ and singular with respect to $(P(t))_{t \geq 0}$ if $\mu \in \mathcal{M}(S)_{\mathrm{TV}}^{S}$. This terminology is based on the fact that $\mu \in \mathcal{M}(S)_{\mathrm{TV}}^{0}$ if and only if there is a $v \in \mathcal{M}(S)_{\mathrm{TV}}^{0}$ such that $\mu \ll|\nu|$ by Theorem 5.7, and $\mu \in \mathcal{M}(S)_{\mathrm{TV}}^{s}$ if and only if $\mu$ and $\nu$ are singular for every $v \in \mathcal{M}(S)_{\mathrm{TV}}^{0}$ by Theorem 5.6.

An immediate consequence of (8) and Corollary 6.3 is the following:

Proposition 6.4 Every $\mu \in \mathcal{M}(S)$ has a unique decomposition $\mu=\mu_{a}+\mu_{s}$, with $\mu_{a} \in \mathcal{M}(S)_{\mathrm{TV}}^{0}$, and $\mu_{s} \in \mathcal{M}(S)_{\mathrm{TV}}^{s}$.

We denote the band projections on $\mathcal{M}(S)_{\mathrm{TV}}^{0}$ and $\mathcal{M}(S)_{\mathrm{TV}}^{s}$ by $P_{0}$ and $P_{S}$ respectively. Then $P_{0}, P_{S}$ are positive bounded linear operators on $\mathcal{M}(S)_{\mathrm{TV}}$, with $\left\|P_{0}\right\| \leq 1$ and $\left\|P_{s}\right\| \leq 1$, and $P_{0} \mu=\mu_{a}, P_{s} \mu=\mu_{s}$.

While $\overline{\mathcal{M}}(S)_{\mathrm{TV}}^{0}$ is invariant under $(P(t))_{t \geq 0}, \mathcal{M}(S)_{\mathrm{TV}}^{s}$ need not be, as the following example shows: Let $S=\mathbb{R}_{+}$with euclidean metric. Define $\Phi_{t}(x)=\max (x-$ $t, 0)$, for $t, x \in \mathbb{R}_{+}$. Then $\left(\Phi_{t}\right)_{t \geq 0}$ is a strongly continuous semigroup of continuous maps on $S$, hence it defines, by Proposition 3.6, a strongly stochastically continuous Markov semigroup $(P(t))_{t \geq 0}$ given by $P(t) \mu:=\mu \circ \Phi_{t}^{-1}$. Let $x>0$, then clearly $\delta_{x} \perp P(t) \delta_{x}$ for all $t>0$, hence $\delta_{x} \in \mathcal{M}(S)_{\mathrm{TV}}^{s}$. However, for $t \geq x, P(t) \delta_{x}=\delta_{0}$, and $\delta_{0}$ is in $\mathcal{M}(S)_{\mathrm{TV}}^{0}$, and not in $\mathcal{M}(S)_{\mathrm{TV}}^{S}$, since $P(t) \delta_{0}=\delta_{0}$ for all $t \in \mathbb{R}_{+}$.

For each $\mu \in \mathcal{M}(S)$, we can define $d\left(\mu, \mathcal{M}(S)_{\mathrm{TV}}^{0}\right)$ to be the distance of $\mu$ to $\mathcal{M}(S)_{\mathrm{TV}}^{0}$ with respect to $\|\cdot\|_{\mathrm{TV}}$. Clearly, $\mu \in \mathcal{M}(S)_{\mathrm{TV}}^{0}$ if and only if $d\left(\mu, \mathcal{M}(S)_{\mathrm{TV}}^{0}\right)=0$.

Lemma 6.5 Let $\mu \in \mathcal{M}(S)$. Then $d\left(\mu, \mathcal{M}(S)_{\mathrm{TV}}^{0}\right)=\left\|\mu_{s}\right\|_{\mathrm{TV}}$.

Proof ' $\leq$ ': $\mu=\mu_{a}+\mu_{s}$, so $\left\|\mu-\mu_{a}\right\|_{\mathrm{TV}}=\left\|\mu_{s}\right\|_{\mathrm{TV}}$. Hence

$$
d\left(\mu, \mathcal{M}(S)_{\mathrm{TV}}^{0}\right)=\inf _{v \in \mathcal{M}(S)_{\mathrm{TV}}^{0}}\|\mu-v\|_{\mathrm{TV}} \leq\left\|\mu-\mu_{a}\right\|_{\mathrm{TV}}=\left\|\mu_{s}\right\|_{\mathrm{TV}}
$$

' $\geq$ ': Let $v \in \mathcal{M}(S)_{\mathrm{TV}}^{0}$. Then

$$
\left\|\mu_{s}\right\|_{\mathrm{TV}}=\left\|P_{s} \mu\right\|_{\mathrm{TV}}=\left\|P_{s} \mu-P_{s} v\right\|_{\mathrm{TV}} \leq\|\mu-v\|_{\mathrm{TV}},
$$

which implies that $\left\|\mu_{s}\right\|_{\mathrm{TV}} \leq d\left(\mu, \mathcal{M}(S)_{\mathrm{TV}}^{0}\right)$.

Lemma 6.6 Let $\mu \in \mathcal{M}(S)$. The function $t \mapsto\left\|P_{S} P(t) \mu\right\|_{\mathrm{TV}}$ is non-increasing.

Proof It suffices to show that $\left\|P_{S} P(t) \mu\right\|_{\mathrm{TV}} \leq\left\|P_{S} \mu\right\|_{\mathrm{TV}}$ for all $t \in \mathbb{R}_{+}$.

Let $0 \leq t$. First assume $\mu \in \mathcal{M}^{+}(S)$, then $0 \leq \mu_{a} \leq \mu$. Since $\mathcal{M}(S)_{\mathrm{TV}}^{0}$ is invariant under $P(t), P_{0} P(t) \mu_{a}=P(t) \mu_{a}$, hence

$$
0 \leq P(t) \mu_{a}=P_{0} P(t) \mu_{a} \leq P_{0} P(t) \mu .
$$

Then

$$
0 \leq P_{s} P(t) \mu=P(t) \mu-P_{0} P(t) \mu \leq P(t) \mu-P(t) \mu_{a}=P(t) \mu_{s},
$$


hence

$$
\left\|P_{S} P(t) \mu\right\|_{\mathrm{TV}} \leq\left\|P(t) \mu_{s}\right\|_{\mathrm{TV}} \leq\left\|\mu_{S}\right\|_{\mathrm{TV}} .
$$

Now let $\mu=\mu^{+}-\mu^{-} \in \mathcal{M}(S)$. Then $P_{s} \mu^{+} \perp P_{s} \mu^{-}$, which implies that $\left\|P_{s} \mu\right\|_{\mathrm{TV}}=\left\|P_{s} \mu^{+}\right\|_{\mathrm{TV}}+\left\|P_{s} \mu^{-}\right\|_{\mathrm{TV}}$. By (9)

$$
\begin{aligned}
\left\|P_{s} P(t) \mu\right\|_{\mathrm{TV}} & \leq\left\|P_{s} P(t) \mu^{+}\right\|_{\mathrm{TV}}+\left\|P_{s} P(t) \mu^{-}\right\|_{\mathrm{TV}} \\
& \leq\left\|P_{s} \mu^{+}\right\|_{\mathrm{TV}}+\left\|P_{s} \mu^{-}\right\|_{\mathrm{TV}}=\left\|P_{s} \mu\right\|_{\mathrm{TV}}
\end{aligned}
$$

\subsection{A Wiener-Young type theorem}

Wiener and Young [30] extended the result by Plessner (see Sect. 6.1), by showing that for all $\mu \in \mathcal{M}(\mathbb{R}), \lim \sup _{t \rightarrow 0}\|P(t) \mu-\mu\|_{\mathrm{TV}}=2\left\|\mu_{s}\right\|_{\mathrm{TV}}$, where $P(t) \mu=\mu \circ$ $\Phi_{t}^{-1}$, with $\Phi_{t}(x)=x+t$, and $\mu_{s}$ is the singular component of $\mu$ with respect to the Lebesgue measure.

We generalise this result to the Markov semigroups with conditions as before. It has been generalised in several other directions: see for instance [24, 25] for a generalisation in the setting of adjoint semigroups of positive strongly continuous semigroups on Banach lattices. Note that the Markov semigroups we consider here are in general not adjoints of strongly continuous semigroups.

Theorem 6.7 Let $\mu \in \mathcal{M}(S)$. Then $\lim \sup _{t \downarrow 0}\|P(t) \mu-\mu\|_{\mathrm{TV}}=2\left\|\mu_{s}\right\|_{\mathrm{TV}}$.

Proof Step 1. $\lim _{t \downarrow 0}\|P(t) \mu\|_{\mathrm{TV}}=\|\mu\|_{\mathrm{TV}}$ for all $\mu \in \mathcal{M}(S)$.

Let $\epsilon>0$. Since

$$
\|\mu\|_{\mathrm{TV}}=\sup \left\{\left|\int_{S} f d \mu\right|: f \in C_{b}(S),\|f\|_{\infty} \leq 1\right\},
$$

there is an $f \in C_{b}(S)$ with $\|f\|_{\infty} \leq 1$ and $\left|\|\mu\|_{\mathrm{TV}}-\int_{S} f d \mu\right|<\frac{\epsilon}{2}$. By strong continuity of $(P(t))_{t \geq 0}$ and Lemma 3.5 there exists a $\tau>0$, such that

$$
|\langle P(t) \mu, f\rangle-\langle\mu, f\rangle|<\frac{\epsilon}{2} \quad \text { for all } t \in[0, \tau) .
$$

Thus for $t \in[0, \tau)$ we obtain

$$
\|P(t) \mu\|_{\mathrm{TV}} \geq|\langle P(t) \mu, f\rangle| \geq\|\mu\|_{\mathrm{TV}}-\epsilon,
$$

and by (MO2) $\|P(t) \mu\|_{\mathrm{TV}} \leq\|\mu\|_{\mathrm{TV}}$, hence the statement holds.

Step 2. $\lim \sup _{t \downarrow 0}\|P(t) \mu-\mu\|_{\mathrm{TV}}=2\left\|\mu_{s}\right\|_{\mathrm{TV}}$ for all $\mu \in \mathcal{M}(S)$.

Clearly $\left\|P(t) \mu_{a}-\mu_{a}\right\|_{\mathrm{TV}} \rightarrow 0$. This implies that $\limsup _{t \downarrow 0}\|P(t) \mu-\mu\|_{\mathrm{TV}}=$ $\limsup \operatorname{su}_{t 0}\left\|P(t) \mu_{s}-\mu_{s}\right\|_{\mathrm{TV}}$. By Proposition 6.2, $P(t) \mu_{s} \perp \mu_{s}$ for almost every $t \in$ $[0, \infty)$, say for all $t \in N$, where $[0, \infty) \backslash N$ has measure zero. Hence, for these $t$, $\left\|P(t) \mu_{s}-\mu_{s}\right\|_{\mathrm{TV}}=\left\|P(t) \mu_{s}\right\|_{\mathrm{TV}}+\left\|\mu_{s}\right\|_{\mathrm{TV}}$ and thus $\left\|P(t) \mu_{s}-\mu_{s}\right\|_{\mathrm{TV}} \rightarrow 2\left\|\mu_{s}\right\|_{\mathrm{TV}}$ as $t \downarrow 0$ in $[0, \infty) \backslash N$ by Step 1 . Noting that $\left\|P(t) \mu_{s}-\mu_{s}\right\|_{\mathrm{TV}} \leq 2\left\|\mu_{s}\right\|_{\mathrm{TV}}$ by the triangle inequality and (MO2), the proof of Step 2 is complete. 
Acknowledgements We thank the referee for suggesting the term "strongly stochastically continuous" Markov semigroup instead of "strongly continuous", which may be too ambiguous. He/she also proposed a simplification in the proof of Proposition 3.6(ii), second part, and provided a shorter version of the proof of Theorem 6.7, Step 2, which is presented above.

Open Access This article is distributed under the terms of the Creative Commons Attribution Noncommercial License which permits any noncommercial use, distribution, and reproduction in any medium, provided the original author(s) and source are credited.

\section{References}

1. Albiac, F., Kalton, N.J.: Topics in Banach Space Theory. Graduate Texts in Mathematics, vol. 233. Springer, Berlin (2006)

2. Aliprantis, C., Burkinshaw, O.: Positive Operators. Academic Press, New York (1985)

3. Billingsley, P.: Convergence of Probability Measures, 2nd edn. Wiley, New York (1999)

4. Conway, J.B.: A Course in Functional Analysis, 2nd edn. Springer, Berlin (1990)

5. Diekmann, O., Gyllenberg, M., Thieme, H.R., Verduyn Lunel, S.M.: A cell-cycle model revisited, Report AM-R9305, Centrum voor Wiskunde en Informatica, Amsterdam (1993)

6. Diekmann, O., Gyllenberg, M., Metz, J.A.J., Thieme, H.R.: On the formulation and analysis of general deterministic structured population models; I. Linear theory. J. Math. Biol. 36, 349-388 (1998)

7. Diekmann, O., Gyllenberg, M., Huang, H., Kirkilionis, M., Metz, J.A.J., Thieme, H.R.: On the formulation and analysis of general deterministic structured population models; II. Nonlinear theory. J. Math. Biol. 43, 157-189 (2001)

8. Diestel, J., Uhl, J.J. Jr.: Vector Measures. Math. Surveys, vol. 15. Am. Math. Soc., Providence (1977)

9. Dorroh, J.R., Neuberger, J.W.: A theory of strongly continuous semigroups in terms of Lie generators. J. Funct. Anal. 136, 114-126 (1996)

10. Dudley, R.M.: Convergence of Baire measures. Stud. Math. 27, 251-268 (1966)

11. Dudley, R.M.: Correction to: "Convergence of Baire measures". Stud. Math. 51, 275 (1974)

12. Engel, K.-J., Nagel, R.: One-Parameter Semigroups for Linear Evolution Equations. Graduate Texts in Mathematics, vol. 194. Springer, Berlin (2000)

13. Ethier, S.N., Kurtz, T.G.: Markov Processes; Characterization and Convergence. Wiley, New York (1986)

14. Feller, W.: An Introduction to Probability Theory and Its Applications, vol. II. Wiley, New York (1965)

15. Gulick, S.L., Liu, T.S., van Rooij, A.C.M.: Group algebra modules II. Canad. J. Math. 19, 151-173 (1967)

16. Hille, E., Phillips, R.S.: Functional Analysis and Semi-groups, revised edn. Colloquium Publications, vol. XXXI. Am. Math Soc., Providence (1957)

17. Hille, S.C.: Continuity of the restriction of $C_{0}$-semigroups to invariant Banach subspaces. Integral Equations Operator Theory 53, 597-601 (2005)

18. Hille, S.C., Worm, D.T.H.: Embedding of semigroups of Lipschitz maps into positive linear semigroups on ordered Banach spaces generated by measures. Integral Equations Operator Theory $\mathbf{6 3}$, 351-371 (2009)

19. Lant, T., Thieme, H.R.: Markov transition functions and semigroups of measures. Semigroup Forum 74, 337-369 (2007)

20. Lasota, A., Mackey, M.C.: Chaos, Fractals, and Noise. Stochastic Aspects of Dynamics, 2nd edn. Springer, New York (1994)

21. Liu, T.S., van Rooij, A.C.M.: Transformation groups and absolutely continuous measures. Indag. Math. 30, 225-231 (1968)

22. Meyer-Nieberg, P.: Banach Lattices. Springer, Berlin (1991)

23. Narici, L., Beckenstein, E.: Topological Vector Spaces. Dekker, New York (1985)

24. van Neerven, J.M.A.M., de Pagter, B.: The adjoint of a positive semigroup. Compos. Math. 90, 99118 (1994)

25. de Pagter, B.: A Wiener-Young type theorem for dual semigroups. Acta Appl. Math. 27, 101-109 (1992) 
26. Plessner, A.: Eine Kennzeichnung der totalstetigen Funktionen. J. Reine Angew. Math. 60, 26-32 (1929)

27. Pichór, K., Rudnicki, R.: Asymptotic behaviour of Markov semigroups and applications to transport equations. Bull. Polish Acad. Math. 45, 379-397 (1997)

28. Pichór, K., Rudnicki, R.: Continuous Markov semigroups and stability of transport equations. J. Math. Anal. Appl. 249, 668-685 (2000)

29. Thieme, H.R., Voigt, J.: Stochastic semigroups: their construction by perturbation and approximation. In: Weber, M.R., Voigt, J. (eds.) Proc.: Positivity IV-Theory and Applications, pp. 135-146. TU Dresden, Dresden (2006)

30. Wiener, N., Young, R.C.: The total variation of $g(x+h)-g(x)$. Trans. Am. Math. Soc. 33, 327-340 (1935)

31. Williams, D.: Diffusions, Markov Processes and Martingales. Volume 1: Foundations. Wiley, Chichester (1979)

32. Zaanen, A.C.: Introduction to Operator Theory in Riesz Spaces. Springer, Berlin (1997) 